\title{
UNSTABLE STRUCTURES DEFINABLE IN O-MINIMAL THEORIES
}

\author{
ASSAF HASSON* AND ALF ONSHUUS
}

\begin{abstract}
Let $\mathcal{M}$ be an o-minimal structure with elimination of imaginaries, $\mathcal{N}$ an unstable structure definable in $\mathcal{M}$. Then there exists $X$, definable in $\mathcal{N}^{e q}$, such that $X$ with all the structure induced from $\mathcal{N}$ is o-minimal. In particular $X$ is linearly ordered.

As part of the proof we show: Theorem 1: If $\operatorname{dim}_{\mathcal{M}} N=1$ then any $p \in S_{1}(N)$ is either strongly stable or finite by o-minimal. Theorem 2: If $N$ is $\mathcal{N}$-minimal then $\operatorname{dim}_{\mathcal{M}} N=1$.
\end{abstract}

\section{BACKGROUND}

1.1. Introduction. Various results in and around o-minimality give hope that some classification of theories interpretable in o-minimal structures may exist. As a first step in that direction Y. Peterzil suggested to examine whether theories interpretable in o-minimal structures admitted some sort of analysis in terms of (b-)minimal types satisfying Zilber's trichotomy.

Zilber's idea of classifying the combinatorial geometries associated with minimal types as trivial, linear or "field like" is an important source of inspiration for model theoretic research. Although not always possible, the search for such a classification has, in many cases, resulted in a deeper understanding of the fine structure of the theories in question. The results of [PS98 and HZ96 leave room for the hope that such a trichotomy may hold for minimal types in theories interpretable in o-minimal structures.

Naturally, any such classification will have to comprise Zilber's trichotomy for minimal stable structures in o-minimal theories, a prospect which seems, at the moment, out of reach. The unstable case, however, seems quite accessible relying on the Trichotomy Theorem for o-minimal structures of [PS98] and Shelah's early analysis of dependent theories.

To tackle the unstable case, Peterzil's suggestion was to prove first that any such structure interprets an o-minimal set. In this paper we carry this out, obtaining somewhat sharper results.

\section{Definition 1.1.}

(1) Let $\mathcal{N}$ be any structure and $X \subseteq N^{n}$ definable. Say that $X$ is finite by o-minimal if there is a definable equivalence relation $E$ with finite

Date: July 24, 2021.

${ }^{*}$ Supported by the EPSRC grant no. EP C52800X 1. 
classes and domain $X$ and a definable linear order $<$ on $X / E$ such that $(X / E,<)$, with all the induced structure from $\mathcal{N}$, is o-minimal.

(2) A type $p$ over $A \subset X$ is finite by o-minimal if it is $p$-minimal (i.e. has $U^{b}$-rank 1, see Definition 4.1) and such that there is a non algebraic extension of $p$ containing a finite by o-minimal formula.

We can now state our main result:

Theorem 1.2. Let $\mathcal{N}$ be definable in an o-minimal structure. Then either there is an $\mathcal{N}$-definable subset of $N$ which is finite by o-minimal or there is an $\mathcal{N}$-definable subset of $N$ which is stable.

Moreover, if $\mathcal{N}$ is $\kappa$-saturated and $\left|N_{0}\right|<\kappa$ for some $N_{0} \subset N$ then every type $p \in S_{1}^{\mathcal{N}}\left(N_{0}\right)$ can be extended to a non algebraic type $q \in S_{1}^{\mathcal{N}}(N)$ which is either finite by o-minimal or strongly stable.

The notion of strongly stable types requires explanation:

Definition 1.3. Let $T$ be a dependent theory, $\mathcal{N} \models T$. A type $p \in S(N)$ is strongly stable if there are no $p^{\prime} \supseteq p$ and formula $\phi(x, y)$ defining a quasi order with infinite chains in $p^{\prime}$.

Strongly stable types are stable according to Shelah's definition of stable types in a dependent theory (see $\S 1$ of [She04]), but the definitions are easily seen not to be equivalent (see the example concluding Section 2). Types which are not strongly stable will be called weakly unstable.

On the global level we can strengthen the result of Theorem 1.2 .

Theorem 1.4. Let $\mathcal{M}:=(M,<, \ldots)$ be an o-minimal structure with a dense underlying order and elimination of imaginaries. It $\mathcal{N}$ is unstable, interpretable in $\mathcal{M}$, then $\mathcal{N}$ interprets an o-minimal structure.

These results, together with the more accurate local statement of the 1dimensional case (Theorem 2.1) and the reduction to it (Section 4, Claim 4.16) give rise to the hope that a structural analysis of types in terms of o-minimal and minimal stable types could be achieved. An obvious obstacle on the way of such a program is the fact that Theorem 1.4 does not have an immediate local analogue (strengthening the second part of Theorem 1.2), as shows an easy example in Section 3. It seems that, in order to formulate (and prove) a correct analogue of that theorem, machinery such as theories of domination, analysability, stable domination and weight may have to be developed. It is not quite clear to us what is the right context for such a project. Recent work of Shelah in She suggests some directions (in the significantly more general setting of dependent theories) which may be of relevance to the present project.

The structure of this paper is as follows. In Section 2 we show that given any 1-dimensional partially ordered set $\left(N, \leq_{p}\right)$ definable in an o-minimal structure $\mathcal{M}$ there is a $\leq_{p}$-definable finite by o-minimal $X \subseteq N$. 
The strategy of the proof of Theorem 1.4 will be to inductively reduce the problem to the 1-dimensional case. In Section 3 we perform the first part of the induction step, showing that if $\mathcal{N}$ is any structure definable in an o-minimal $\mathcal{M}$, then $N$ is $\mathcal{N}$-minimal only if it is either strongly minimal or 1-dimensional. In other words if $\mathcal{N}$ is unstable and $\operatorname{dim} N>1$ there exists an $\mathcal{N}$-definable $X \subseteq N$ with $\operatorname{dim} X<\operatorname{dim} N$.

The induction is completed in section 4, where Theorem 1.4 is proved using the machinery of p-forking. The proof consists of showing that, assuming elimination of imaginaries in the ambient o-minimal structure, we can actually find a lower dimensional unstable set.

1.2. Dependent theories (or theories with NIP). We assume the reader has certain familiarity with basic notions of model theory, o-minimality and geometric structures. We list some known facts which will be used repeatedly throughout the paper and refer to vdD98] and [Pil96] for the necessary background in o-minimality and stability respectively.

We begin with:

Definition 1.5. Let $T$ be any theory and $\mathcal{C} \models T$ a monster model.

(1) a formula $\phi(x, y)$ has the order property if there are indiscernible sequences $\left\langle a_{i}\right\rangle_{i \in \omega}$ and $\left\langle b_{i}\right\rangle_{i \in \omega}$ such that $\mathcal{C} \models \phi\left(a_{i}, b_{j}\right)$ if and only if $i<j$.

(2) A formula $\phi(x, y)$ has the strict order property if there is an indiscernible sequences $\left\langle a_{i}\right\rangle_{i \in \omega}$ such that $\mathcal{C} \models \exists y \phi\left(y, a_{i}\right) \wedge \neg \phi\left(y, a_{j}\right)$ if and only if $i<j$.

(3) A formula $\phi(x, y)$ has the independence property if there is an indiscernible sequences $\left\langle a_{i}\right\rangle_{i \in \omega}$ such that for any finite disjoint sets $I$ and $J$ there is some $c$ such that $\mathcal{C} \models \phi\left(c, a_{i}\right)$ for any $i \in I$ and $\mathcal{C} \models \neg \phi\left(y, a_{j}\right)$ whenever $j \in J$.

(4) A theory $T$ is dependent(equivalently, does not have the independence property, or has the non-independence property) if no formula has the independence property.

(5) A definable set $\theta(x)$ is stable if there is no $\phi(x, y) \in \mathcal{C}$ such that $\phi(x, y) \wedge \theta(x)$ has the order property.

The following theorem is a rehash of results from [She04 and [She90] presented more conveniently for our needs in $\mathrm{OP}$.

Theorem 1.6. Let $X=X(\mathcal{C})$ be a set interpretable in a dependent theory. The following are equivalent:

- $X:=\theta(x)$ is an unstable set.

- There exists a formula $\phi(x, y)$ such that $\phi(x, y) \wedge \theta(x)$ has the order property.

- There are sequences $\left\langle a_{i}\right\rangle_{i \in \omega}$ and $\left\langle b_{i}\right\rangle_{i \in \omega}$ such that $a_{i} \in X(\mathcal{C})$ for all $i, b_{j} \in \mathcal{C}$ for all $j$ and $\mathcal{C} \models \phi\left(a_{i}, b_{j}\right)$ if and only if $i<j$.

- There is a $\mathcal{C}$-definable partial order on $X(\mathcal{C})$ with infinite chains. 
Given a dependent structure $\mathcal{N}$ we will say that an $\mathcal{N}$-definable set $X$ is stable if it satisfies any of the above conditions.

As an easy corollary of Theorem 1.6 we have the following theorem which is the technical basis of this paper.

Theorem 1.7 (Shelah). Let $\mathcal{N}$ be any structure interpretable in an o-minimal theory $T$. Then for any $\mathcal{N}$-definable unstable $X \subseteq N^{k}$ there is an $\mathcal{N}$-definable partial quasi order $\leq_{p}$ on $X$ with infinite chains.

Proof. Since $\mathcal{N}$ is interpretable in an o-minimal theory it does not have the independence property, and neither does $X$ with all the induced structure. The result now follows from Theorem 1.6.

We conclude with some notational conventions that will be kept throughout the paper. $\mathcal{M}:=(M,<, \ldots)$ will always denote an o-minimal structure with a dense underlying order. $\mathcal{N}$ will be a structure definable in $\mathcal{M}$ (in other words, given a structure $\mathcal{N}$ definable in some o-minimal structure, we fix such a structure $\mathcal{M}$ and an interpretation of $\mathcal{N}$ therein). Given any $\mathcal{N}$ definable $X \subseteq N$ the dimension of $X$, denoted $\operatorname{dim}(X)$, is the o-minimal dimension of $X$ as an $\mathcal{M}$-definable set.

\section{The 1-Dimensional CASE}

Theorem 2.1. Let $\mathcal{M}:=(M,<, \ldots)$ be a dense o-minimal structure, $X \subset$ $M$ an infinite definable set and $\leq_{p}$ an $\mathcal{M}$-definable order with infinite chains on $X$. Let $p \in S_{1}(M)$ be a type extending $x \in X$ with infinite $\lessgtr_{p}$-chains.

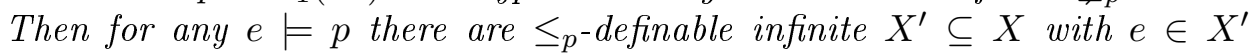
generic and a linear order $<^{\prime}$ on $X^{\prime}$ such that $\left(X^{\prime},<^{\prime}\right) \equiv\left(X^{\prime},<\right)$.

Some conventions regarding terminology are in place. As in the statement of the theorem, a set definable in $\left(X, \leq_{p}\right)$ will be called $\leq_{p}$-definable (or $X$ definable). The term "definable set" will always refer to $\mathcal{M}$-definable sets. All orders will be partial, unless explicitly stated. Hence a quasi order is a transitive binary relation $\leq$. A quasi order is said to have infinite chains if the corresponding order (obtained after quotienting by the equivalence relation $a \leq b \wedge b \leq a$ ) does. Throughout the text all (quasi) orders will be assumed to have infinite chains. We will also assume, without loss of generality, that $\mathcal{M}$ is saturated enough (so that every $\mathcal{M}$-definable set has a generic point in $\mathcal{M}$ ).

For $x \in X$ it will be convenient to denote $G(x):=\left\{y \mid x \leq_{p} y\right\}$ and $L(x):=\left\{y \mid y \leq_{p} x\right\}$.

The first part of the proof is to decompose $X$ into well behaved cells and redefine the partial order to obtain topologically nice upper and lower cones. Getting the right decomposition of $X$ and the right partial order to work with are the main parts of the proof. 
2.1. Taming $\left(X, \leq_{p}\right)$. By o-minimality, for every $a \in X$, we can write $G(a)=\bigcup_{i=1}^{k} I_{i}$ where each $I_{i}$ is a definably connected component of $G(a)$ and $I_{i} \leq I_{j} \Longleftrightarrow i \leq j$. Let $f_{j}^{1}(a)$ and $f_{j}^{2}(a)$ be the left and right endpoints of $I_{j}(a)$ (which coincide if $I_{j}(a)$ is a point). By allowing empty intervals, we may assume that $k$ does not depend on $a$ (since the number of connected definably components of $G(a)$ is uniformly bounded).

Let $X_{1}, \ldots, X_{n}$ be a decomposition of $X$ such that all the $f_{j}^{i}$ are continuous on each $X_{s}$ and such that each $f_{j}^{i}$ is either non-increasing or non-decreasing in $X_{k}$ for all $k$. With this decomposition of $X$ we redefine the intervals $I_{j}(a)$ to make sure that $I_{j}(a)$ is entirely contained in some $X_{i}$ for all $a$ and $i$. This can be done as follows: for each $a \in X$ and $i \leq k$ define $I_{j}^{i}(a)=I_{j}(a) \cap X_{i}$. The functions that define our new intervals will be (weakly) monotone and continuous.

In order to formalise this (and since we will repeat the same process over and over again in this section), we need the following definitions.

Definition 2.2. Let $\mathcal{M}$ be an o-minimal structure, $X \subseteq M$ an infinite definable subset and $\leq_{p}$ an $\mathcal{M}$-definable relation inducing a partial order on $X$. Denote $\mathcal{M}_{\infty}$ the natural expansion of $\mathcal{M}$ to $M \cup\{ \pm \infty\}$. A monotone representation of $\left(X, \leq_{p}\right)$ is a decomposition $X=\bigcup_{i=1}^{l} X_{i}$ into disjoint $\mathcal{M}$ definable intervals and points, and two finite collections of definable functions

$$
\mathcal{F}^{G}:\left\{f_{j}^{i}\left|X \rightarrow \operatorname{cl}_{\mathcal{M}_{\infty}}(X)\right| 1 \leq j \leq n, i \in\{1,2\}\right\}
$$

and

$$
\mathcal{F}^{L}:\left\{h_{j}^{i}\left|X \rightarrow \operatorname{cl}_{\mathcal{M}_{\infty}}(X)\right| 1 \leq j \leq n, i \in\{1,2\}\right\}
$$

with

$$
G(x)=\bigcup_{f_{j}^{i} \in \mathcal{F}^{G}}\left(f_{j}^{1}(x), f_{j}^{2}(x)\right)
$$

and

$$
L(x)=\bigcup_{h_{j}^{i} \in \mathcal{F}^{L}}\left(h_{j}^{1}(x), h_{j}^{2}(x)\right)
$$

for all $x \in X$ and such that:

(1) For every $l \leq k, j \leq n$ and $i \in\{1,2\}$ the function $\left.f_{j}^{i}\right|_{X_{k}}$ is continuous and weakly monotone.

(2) For every $1 \leq j<n$ we have $f_{j}^{1} \leq f_{j}^{2} \leq f_{j+1}^{1}$.

(3) For all $x \in X$ and all $j$ there exists $r$ such that $\left(f_{j}^{1}(x), f_{j}^{2}(x)\right) \subseteq X_{r}$. and analogous conditions (1'), (2') and (3') for $\mathcal{F}^{L}$.

Claim 2.3. Let $\mathcal{M}$ be an o-minimal structure. Then given any 1-dimensional $\mathcal{M}$-definable partial order $\left(X, \leq_{p}\right)$ and any decomposition $\left\{X_{i}\right\}$ of $X$ there is a monotone representation $\left\{X_{i}^{\prime}, \mathcal{F}^{G}, \mathcal{F}^{L}\right\}$ of $\left(X, \leq_{p}\right)$ such that $\left\{X_{i}^{\prime}\right\}$ refines $\left\{X_{i}\right\}$. 
Proof. We start with the given decomposition $\left\{X_{i}\right\}$ of $X$ and we refine it as described before Definition 2.2 to a decomposition $\left\{X_{i}^{\prime}\right\}$ so that all the functions $f(x)$ defining the endpoints of the intervals in both $G(x)$ and $L(x)$ are either non-increasing or non-decreasing in $X_{k}^{\prime}$ for all $k$.

We now define the functions $\left\{f_{j}^{i}\right\}$ which will define the endpoints of the "truncated" intervals (so that each interval composing $G(a)$ is entirely contained in a single cell $\left.X_{i}\right)$.

Let $i_{1}(a):=\min \left\{i \mid I_{i}(a) \neq \emptyset\right\}$ and $i_{2}:=\max \left\{i \mid I_{i}(a) \neq \emptyset\right\}$ and define $f_{j, i_{1}}^{1}(a)=f_{j}^{1}(a)$ and $f_{j, i_{2}}^{2}(a)=f_{j}^{2}(a)$.

For $i_{1}<i<i_{2}$ define

$$
f_{j, i}^{1}(a)=\max \left\{f_{j}^{1}(a), \inf X_{i}^{\prime}\right\}
$$

and

$$
f_{j, i}^{2}(a)=\min \left\{f_{j}^{2}(a), \sup X_{i}\right\}
$$

let

$$
\mathcal{F}^{G}:=\left\{f_{j, i}^{1}\right\}_{i, j} \cup\left\{f_{j, i}^{2}\right\}_{i, j}
$$

Define $\mathcal{F}^{L}$ in a similar way. We leave it as a simple exercise to verify that all the resulting functions are continuous and weakly monotone on each $X_{i}^{\prime}$ so that the collections $\mathcal{F}^{G}, \mathcal{F}^{L}$ satisfy Definition 2.2 with respect to the decomposition $\left\{X_{i}^{\prime}\right\}$, which finishes the proof.

Remark 2.4. To avoid unpleasant trivial cases, after fixing a monotone representation, we will throw away all the $X_{i}$ in the representation consisting of a single point. Replacing $X$ with $\bigcup\left\{X_{i} \mid X_{i}\right.$ is not a point $\}$ we may assume that the monotone representation we are working with consists of open intervals only.

To simplify the notation, when referring to a monotone representation, we will only mention the decomposition of $X$ and use $\mathcal{F}$ to denote $\mathcal{F}^{G} \cup \mathcal{F}^{L}$ whenever no ambiguity can arise.

From now on we fix a monotone representation $\left\{X_{1}, \ldots X_{k}, \mathcal{F}\right\}$ of $\left(X, \leq_{p}\right)$. To keep the exposition cleaner, although we will repeatedly refine it (as explained in Claim 2.3), we will not change the notation for the representation. Our first task is to smoothen up $\leq_{p}$, in order to make it easier to handle.

By o-minimality the relation

$$
a \leq_{p}^{0} b \Leftrightarrow \operatorname{dim}(G(b) \backslash G(a))=0 .
$$

is $\leq_{p}$-definable. Thus

\section{Lemma 2.5.}

(1) $\leq_{p}^{0}$ is a quasi order refining $\leq_{p}$ and every definable $Y \subseteq X$ with infinite $\leq_{p}$-chains contains infinite $\leq_{p}^{0}$-chains. 
(2) For every $a \in X$ let $G^{0}(a):=\left\{x \mid a \leq_{p}^{0} x\right\}$ and $L^{0}(a):=\left\{x \mid x \leq_{p}^{0}\right.$ $a\}$. Then the sets $G^{0}(a) \cap X_{i}$ and $L^{0}(a) \cap X_{i}$ are both relatively closed in $X_{i}$.

Proof. Since $a \leq_{p} b \Rightarrow a \leq_{p}^{0} b$ and $\leq_{p}^{0}$ is transitive it is a definable quasi order refining $\leq_{p}$. By compactness, if $\leq_{p}$ had infinite chains then so will $\leq_{p}^{0}$.

Let $b \in X_{i}$ and let $a \in X$ be such that $a \not_{p}^{0} b$, i.e. $|G(b) \backslash G(a)|$ is infinite, so it contains an interval. The functions in $\mathcal{F}$ are continuous at $b$, so there is an interval $U \subseteq X_{i}$ with $b \in U$ such that $|G(x) \backslash G(a)|$ is infinite (so $a \mathbb{Z}_{p}^{0} x$ ) for all $x \in U$. Therefore $b \notin\left\{x \mid a \leq_{p}^{0} x\right\}$ implies that $b \notin \partial\left\{x \mid a \leq_{p}^{0} x\right\}$, proving that $G^{0}(a)$ is relatively closed in $X_{i}$.

Now suppose that $a \in X_{j}$ and that $b \in X$ is such that $a \not_{p}^{0} b$ so that $\mid G(b) \backslash$ $G(a) \mid$ is infinite, so it contains an open interval $I$. Since $I \cap G(a)=\emptyset$ the continuity of the functions in $\mathcal{F}$ (around $a$ this time) there is a neighbourhood $V$ of $a$ such that $I \backslash G\left(a^{\prime}\right)$ is infinite for all $a^{\prime} \in V$; so $a^{\prime} \not_{p}^{0} b$ for any such $a^{\prime}$ implying, as above, that $L(b)$ is relatively closed in $X_{j}$.

By Claim 2.3 we can find a monotone representation of $\left(X, \leq_{p}^{0}\right)$ refining the monotone representation of $\left(X, \leq_{p}\right)$. So we may assume that $\left(X_{1}, \ldots, X_{k}, \mathcal{F}\right)$ is a monotone representation of both quasi orders. Since Lemma 2.5 is weakened by the refinement of the monotone representation (there are fewer interior points), its conclusion will remain valid as we will further refine $\left(X_{1}, \ldots, X_{k}, \mathcal{F}\right)$.

It may be worth pointing out that even if $\leq_{p}$ is an order, $\leq_{p}^{0}$ need not be one (i.e. it may be a quasi order). But after reducing ourselves to a definable subset of $X$ we may assume that $E(a, b):=a \leq_{p}^{0} b \wedge b \leq_{p}^{0} a$ has finite classes. Thus, identifying each $E$-class with its smallest element we may assume the map $\pi: X \rightarrow X / E$ is in fact a map from $M$ to $M$. Refining the above monotone representation further, we may assume that $\pi$ is continuous on all the cells of the representation. In particular we may assume that $\leq_{p}^{0}$ is in fact an order, and we can work with $\leq_{p}^{0}$ instead of $\leq_{p}$, obtaining the following. The fact that the order $\leq_{p}^{0}$ induces on $X / E$ still satisfies Lemma 2.5 (after possibly removing from $X$ finitely many points) is easy. We obtained:

Fact 2.6. We may assume without loss of generality that $\leq_{p}$ is an order such that the sets $G(a) \cap X_{i}$ and $L(a) \cap X_{i}$ are both relatively closed in $X_{i}$ for every $i$ and every $a \in X$.

One reason for this additional massaging of our monotone representation is to obtain:

Corollary 2.7. If $f_{j}^{2}(x)=f_{j+1}^{1}(x)$ for some $j<|\mathcal{F} / 2|$ and $x \in X_{i}$ (some i) then both functions are locally constant near $x$.

The proof is immediate from the assumptions of Fact 2.6 and we leave it as an easy exercise to the reader. Theorem 2.1 is now proved in two steps. First, we show that it is enough to find an $\mathcal{M}$-definable interval where $\leq_{p}$ agrees with $<$ (the order on $\mathcal{M}$ ), and then we proceed to find such an $\mathcal{M}$-interval. 
2.2. A special case. In this subsection we show that if $\leq_{p}$ agrees with $<$ on some $\mathcal{M}$-definable interval $X_{0}$ then a local version of Theorem 2.1 follows.

Lemma 2.8. Let $\left(X, \leq_{p}\right)$ be an order definable in an o-minimal structure $\mathcal{M}:=(M,<, \ldots)$. Assume that $\operatorname{dim}_{\mathcal{M}} X=1$ and $X=X_{0} \cup Y$ for some $\mathcal{M}$-definable $X_{0}$ such that $\leq\left._{p}\right|_{X_{0}}$ is a dense linear order. Then for any $\mathcal{M}$-generic $e \in X_{0}$ there exists an infinite $\leq_{p}$-definable set $X^{\prime}$ with $e$ in the interior of $X^{\prime}$ such that either $\leq\left._{p}\right|_{X^{\prime}}=\leq\left.\right|_{X^{\prime}}$ or $\geq||_{X^{\prime}}=\leq\left._{p}\right|_{X^{\prime}}$.

Proof. Since $X$ is 1-dimensional, we may assume that $X \subseteq M$. Fix a monotone representation $\left(X_{1}, \ldots, X_{k}, \mathcal{F}\right)$ of $X$, and let $e$ be any $\mathcal{M}$-generic element of $X_{0}$. In the proof we will keep shrinking $X_{0}$, making sure that $e$ is still generic in the subset of $X_{0}$ that we keep. Refining our monotone representation, we may assume that $X_{0}$ is one of the cells in the decomposition of $X$.

Reducing $X_{0}$ if needed and possibly replacing $\leq_{p}(x, y)$ with $\leq_{p}(y, x)$ we may assume, by o-minimality, that $\leq_{p}$ agrees with $<$ on $X_{0}$. Because $\mathcal{F}$ is a finite collection of functions, we reduce $X_{0}$ further to assure that $f^{-1}(c)$ is finite for all $c \in X_{0}$ and $f \in \mathcal{F}$. Note that this can be done without harming any of the previous requirements.

Choose $a<b \in X_{0}$ generic enough and close to each other such that $e \in(a, b)_{\leq_{p}}$. From now on, we will restrict ourselves to the set $Z_{0}:=(a, b)_{\leq_{p}}$. For $x \in Z_{0}$ denote $u(x)=\inf \left\{G(x) \cap X_{0}\right\}$ and $m(x):=\sup \left\{L(x) \cap X_{0}\right\}$.

Fix some generic $x \in Z_{0}$. By assumption (Fact 2.6) we know that $m(x) \leq_{p} x \leq_{p} u(x)$ and by Fact $2.6 m(x)<u(x)$ for all $x \in\left((a, b)_{\leq_{p}} \backslash X_{0}\right)$ (otherwise, $m(x) \leq_{p} x \leq_{p} m(x)$ contradicting the assumption that $x$ is an order). We now refine our monotone representation one last time to assure that $m(x), u(x)$ are continuous and monotone on each cell of the representation; the assumption that $\operatorname{dim} f^{-1}(c)=0$ for all $f \in \mathcal{F}$ and all $c \in X_{0}$ assures that both functions will be in fact strictly monotone.

Let $\mathcal{Z}$ be the collection of infinite $\leq_{p}$-definable subsets of $Z_{0}$ whose interior contains $e$. The proof proceeds by induction on the possible cardinalities of the set

$$
\left\{i \mid\left\{Z \cap X_{i}\right\} \text { is an infinite set. }\right\}
$$

for $Z \in \mathcal{Z}$ (and a fixed decomposition $\left\{X_{i}\right\}$ of $X$ satisfying all the assumptions mentioned up to this point).

Clearly, $\left|Z \cap X_{0}\right|=\infty$ for any $Z \in \mathcal{Z}$ and if for some such $Z$ we get $\operatorname{dim}\left(Z \backslash X_{0}\right)=0$ then $\leq_{p}$ is a linear order on $Z$ (possibly removing finitely many accidental points) and the lemma will follow; thus, we may assume by way of contradiction that any $Z \in \mathcal{Z}$ has infinite intersection with some $X_{i}$. Let $Z \in \mathcal{Z}$ minimise the number of intervals $X_{i}$ with which it has an infinite intersection. For simplicity assume that $Z \cap X_{1}$ is infinite.

Let $c \in Z \cap X_{1}$ be generic. Since $Z_{c}^{\prime}:=(m(c), u(c))_{\leq_{p}}$ contains the $X_{0^{-}}$ subinterval $(m(c), u(c))$ it is an infinite set containing $c$. If $e \notin(m(c), u(c))$ for all generic $c \in Z \cap X_{1}$, then by the continuity of $u(x), m(x)$ we know that 
either $e>u(c)$ for all but finitely many $c \in Z \cap X_{1}$ or $e<m(c)$ for all such $c$. Both cases are analogous so we may assume the latter holds for all generic $c$. We define $Z^{\prime}:=Z \cap\left(a, b^{\prime}\right)_{\leq_{p}}$ for some $a<e<b^{\prime} \leq \inf \left\{m(c) \mid c \in X_{1} \cap Z_{0}\right\}$; by definition $Z^{\prime} \cap X_{1}$ contains no generic points so by continuity $Z^{\prime} \cap X_{1}=\emptyset$ and $e$ is in the interior of $Z^{\prime}$. Since $Z^{\prime} \subseteq Z$ this contradicts the choice of $Z$. Therefore we may assume that $e \in(m(c), u(c))$ for some generic $c \in Z \cap X_{1}$.

We will investigate two cases. Suppose first that $u(x), m(x)$ are both increasing on $X_{1}$ (the case they are both decreasing is similar). This implies that $y \notin(m(c), u(c))_{\leq_{p}}$ for all $y \in X_{1}$ generic over $c$ (for if $y<c$ then $m(y)<m(c)$ implying - by the definition of $m(y)$ - that $y \notin G(m(c))$ and if $y>c$ then $u(y)>u(c)$ and $y \notin L(u(c)))$. Since $Z_{c}:=(m(c), u(c))_{\leq_{p}} \in \mathcal{Z}$, this would lead to a contradiction to the choice of $Z$.

The only remaining possibility is that $m(x)$ is increasing and $u(x)$ is decreasing in $X_{1}$, or vice versa. Consider $m:=\sup \left\{m(x) \mid x \in X_{1} \cap Z\right\}$. If $m<e$ then by restricting ourselves (as above) to $\left(a^{\prime}, b\right)_{\leq_{p}}$ for some $m<a^{\prime}<e$ we get a contradiction to the choice of $Z$ (as we did there). Otherwise (because $e$ is generic) we know that $m>e$. By symmetry we may assume that $u<e$ where $u:=\inf \left\{u(x) \mid x \in X_{1} \cap Z\right\}$. By continuity and monotonicity, this means that $G(x) \cap L(x) \cap X_{0} \neq \emptyset$ for some $x \in X_{1} \cap Z$. Since this is impossible, the lemma follows.

Note that for every generic $e \in X_{0}$ the set $X_{e}^{\prime}$ we found satisfying the conclusion of Lemma 2.8 was defined using one of finitely many formulae $\psi_{1}(x, e), \ldots, \psi_{s}(x, e)$ (depending, possibly, on parameters independent from $e$, on the monotone representation, but not on $e$ itself). So Lemma 2.8 shows that the formula $\theta(z)$ given by the disjunction of the formulae " $\leq_{p}$ restricted to $\psi_{i}(x, z)$ is a dense linear order" is satisfied by every generic $e \in X_{0}$, whence it is true of all but possibly finitely many $e \in X_{0}$.

2.3. Reducing to the special case. We will now show how to obtain the assumptions of the previous subsection and apply the result to prove Theorem 2.1.

Claim 2.9. Let $X_{k}$ be a cell in the representation of $X$. Assume there are $a, b \in X_{k}$ such that $a \leq_{p} b$ and $a<b$ (the case $b<a$ will have analogous results).

(1) If $f_{i}^{1}$ is non-increasing in $X_{k}$ then so is $f_{i}^{2}$ and if $f_{i}^{2}$ is non-decreasing in $X_{k}$ then so is $f_{i}^{1}$; either of these cases implies that $I_{i}(a) \cap I_{i}(b)=\emptyset$.

(2) If $f_{i}^{1}$ is increasing or constant in $X_{k}$ and $f_{i}^{2}$ is decreasing or constant, then $I_{i}(a) \subseteq I_{i}(b)$.

Proof. The first assertions follow from the fact that, since $\leq_{p}$ is a partial order, $G(a) \supset G(b)$ and $I_{i}(a)$ and $I_{k}(a)$ are always disjoint intervals by construction, $I_{j}(b)$ must be contained entirely in a single interval $I_{k}(a)$.

Either of the first two conditions imply that $j \neq k$ and the corresponding conclusions follow. The last assertion is immediate. 
Using the claim, whenever $X_{k}$ satisfies the the assumptions of the claim and $x \in X_{k}$ is generic we can partition the set indexing $\mathcal{F}^{G}$ as follows:

- $J_{1}^{k}$ is the set of integers $i$ such that both $f_{i}^{1}$ and $f_{i}^{2}$ are non-increasing in $X_{k}$ or both are constant.

- $J_{2}^{k}$ is the set of integers for which both $f_{i}^{1}$ and $f_{i}^{2}$ non-decreasing in $X_{k}$ but not both are constant.

- $J_{3}^{k}$ is the elements not in $J_{k}^{1}$ nor in $J_{k}^{2}$.

We are now ready to conclude the proof of the theorem.

Proof of Theorem 2.1. Let $p$ be any $1-\mathcal{N}$-type with infinite chains. We have to show that every $e \models p$ is contained in a finite by o-minimal set. Because the $X_{i}$ in the decomposition of $X$ are $\mathcal{M}$-definable all realizations of $p$ are in the same $X_{i}$; consequently $X_{i}$ has infinite $\leq{ }_{p}$-chains. Therefore, without loss of generality, $X_{i}$ satisfies the assumptions of Claim 2.9. for simplicity assume $X_{i}=X_{1}$.

By the continuity of the functions in $\mathcal{F}$ and using Corollary 2.7, for generic $a \in X_{1}$ there exists $a_{\epsilon}>a$ with $a_{\epsilon} \in X_{1}$ such that for all $a^{\prime} \in\left(a, a_{\epsilon}\right) \cap X_{1}$ we have

$$
G(a) \cap G\left(a^{\prime}\right)=\bigcup_{i} I_{i}(a) \cap I_{i}\left(a^{\prime}\right)
$$

where $I_{i}(a)=\left(f_{i}^{1}(a), f_{i}^{2}(a)\right)$.

Moreover, keeping the decomposition of the index set of $\mathcal{F}^{G}$ obtained above, we get that such $a<a^{\prime}$ satisfy:

$$
G(a) \cap G\left(a^{\prime}\right):=\bigcup_{i \in J_{1}}\left(f_{i}^{1}(a), f_{i}^{2}(x)\right) \cup \bigcup_{i \in J_{2}}\left(f_{i}^{1}(x), f_{i}^{2}(a)\right) \cup \bigcup_{i \in J_{3}}\left(f_{i}^{1}\left(a^{\prime}\right), f_{i}^{2}\left(a^{\prime}\right)\right)
$$

and the definition of the $J_{i}$ implies that $G(a) \cap G\left(a^{\prime}\right) \supseteq G(a) \cap G\left(a^{\prime \prime}\right)$ if and only if and only if $a^{\prime}<a^{\prime \prime}$ for all $a<a^{\prime}<a^{\prime \prime}<a_{\epsilon}$ with $a^{\prime}, a^{\prime \prime} \in X_{1}$.

Setting $x<^{\prime} y$ if and only if $G(a) \cap G(x) \supseteq G(a) \cap G(y)$ we know that $<^{\prime}$ is a $\leq_{p}$-definable quasi order agreeing with $<$ on $\left(a, a_{\epsilon}\right) \cap X_{1}$. So the theorem now follows from Lemma 2.8 and the fact that the linear order $<^{\prime}$ can be uniformly defined in a set containing any generic $e \in X$ such that $e \in X_{k}$ for some $X_{k}$ a cell in the representation of $X$ containing infinite $\leq_{p}$-chains.

2.4. Further remarks. We conclude with the following observation:

Definition 2.10. A structure $\mathcal{N}$ is definable in an o-minimal structure $\mathcal{M}$, if it is interpretable in the real sort of $\mathcal{M}$ (i.e. the universe of the underlying interpretation is definable).

We will define $N$ to be $k$-dimensional if $k$ is the smallest integer such that there exists an o-minimal structure $\mathcal{M}$ and a definable $S \subseteq M^{r}$ with $\operatorname{dim}_{\mathcal{M}} S=k$ such that there is an interpretation of $\mathcal{N}($ coming from $\mathcal{M})$ with universe $S$. 
Corollary 2.11. Let $\mathcal{N}$ be an unstable structure definable in an o-minimal structure, and let $\mathcal{M}$ witness that $\mathcal{N}$ is 1-dimensional. Then $\mathcal{N}$ interprets an o-minimal structure definable in $\mathcal{M}$.

Proof. Since $\mathcal{N}$ is definable in an o-minimal structure it does not have the independence property. Hence, by theorem 1.7 there is a definable quasi order with infinite chains whose universe is (a subset of) $N$. Let $a \sim b \Longleftrightarrow$ $a \leq_{p} b \wedge b \leq_{p} a$. By o-minimality and the fact that $\leq_{p}$ has infinite chains, we get that $\sim$ has only finitely many infinite classes (and infinitely many finite classes). Passing to the definable subset $N^{\prime}$ of those elements whose $\sim$-classes are finite the structure $N / \sim$ is definable in the same o-minimal structure. The conclusion now follows from Theorem 2.1.

Remark 2.12. It should probably be pointed out that Theorem 1.2 cannot be strengthened to assure that given a (weakly) unstable type $p$ (i.e. a type that is not strongly stable) there is a definable set contained in $p$ which is finite by o-minimal. The following example is due to Kobi Peterzil. Consider the structure $\mathcal{R}$ consisting of the (unordered) group $(\mathbb{R},+)$ expanded by a predicate for the interval $[0,1]$. For $0 \leq r, t \leq n \in \mathbb{N}$ the formula $\psi_{n}(r, t):=$ $\forall z(z+r \in[0, n] \rightarrow z+t \in[0,1])$ defines a linear order on $[0, n]$. Using a simple quantifier elimination argument it is not hard to verify that no unbounded linear order is definable in this structure. Now consider the type $p:=\left\{\neg\left(\psi_{n}(x-r, 0) \vee \psi_{n}(0, x-r)\right) \mid r \in \mathbb{R}\right\} . p$ is weakly unstable, but no formula in $p$ is finite by o-minimal.

Note, however, that $p$ is a stable type according to Shelah (any Morley sequence in $p$ - this is well defined because $p$ is definable - is an indiscernible set). More specifically, denote $\leq_{n}$ the order on $[-n, n]$ defined above. Take $\left\{a_{i}\right\}_{i \in \omega}$ such that $a_{0}=0, a_{i}-a \mathbb{Z}_{n} 0$ for all $a \in \mathbb{R}\left\langle a_{1}, \ldots, a_{i-1}\right\rangle$ (the group generated by $\mathbb{R}$ and $\left.a_{1}, \ldots, a_{i-1}\right)$. It is not hard to verify that $\left\{a_{i}\right\}_{i>0}$ is an indiscernible set, and witnesses the stability of $p$. Note that $p$ does not have U-rank, since every non algebraic forking extension thereof is unstable. It is, however, regular and locally modular.

\section{Finding a Set of SMaller Dimension.}

Keeping in mind the goal of interpreting an o-minimal order in any unstable structure definable in an o-minimal theory, it is natural to pursue an inductive argument based on the o-minimal dimension of the interpretation. Having proved the desired result for the 1-dimensional case, the next step is, given an $n$-dimensional $\mathcal{N}$, definable in an o-minimal structure $\mathcal{M}$, to find an $\mathcal{N}$-definable $X \subseteq N$ with $\operatorname{dim}_{\mathcal{M}} X<n$. This is the goal of the present section.

Let $\mathcal{N}, \mathcal{M}$ be as above with $N \subseteq M^{k}$. By Shelah's theorem (Theorem 1.7) there is an $\mathcal{N}$-definable quasi order $\leq_{p}$ with infinite chains on $N$. For simplicity we may assume that $\mathcal{N}=\left(N, \leq_{p}\right)$. We will show that if $N$ is $\mathcal{N}$-minimal, i.e. every non-algebraic $\mathcal{N}$-definable subset of $N$ has dimension $n$, then $n=1$. 
As the next remark shows, if $N$ is $\mathcal{N}$-minimal it has an intrinsic notion of dimension. To avoid confusion the use of the term "dimension" will be reserved exclusively for the o-minimal dimension, and "generic" will always mean "M-generic" (over the relevant data). To simplify things, we may assume that $N$ and $\leq_{p}$ are $\emptyset$-definable in $\mathcal{M}$. The following appears already in [PPS00], but we give the simple proof:

Remark 3.1. Let $\mathcal{N}$ be a structure definable in an o-minimal structure $\mathcal{M}$. If for all $a \in N$ either $\operatorname{tp}(a / A)$ is algebraic or $\operatorname{dim}_{\mathcal{M}}(\operatorname{tp}(a / A))=n$ then $\mathcal{N}$ is a geometric structure, i.e. the model theoretic algebraic closure acl $\mathcal{N}_{\mathcal{N}}(\cdot)$ satisfies the Exchange Property and $\mathcal{N}$ eliminates the quantifier $\exists^{\infty}$.

Proof. Everything is clear, except exchange. So let $a \in \operatorname{acl}(A b) \backslash \operatorname{acl}(A)$. We have to show that $b \in \operatorname{acl}(A a)$. If $\operatorname{dim} \operatorname{tp}(b / A a)<n$ it must be algebraic and the proposition follows. Otherwise, possibly replacing $b$ we may assume that $\operatorname{dim}(b / A a)=n$. Since $a \notin \operatorname{acl}(A)$ we know that $a \bigotimes_{A} b$ (in the ominimal sense). Therefore, $b \bigsqcup_{A} a$ implying that $\operatorname{dim} \operatorname{tp}_{\mathcal{M}}(b / A a)<n$ which contradicts our assumptions.

Throughout this section we will assume that $N$ is $\mathcal{N}$-minimal, so in particular every infinite $\mathcal{N}$-definable $S \subseteq N$ contains an $\mathcal{M}$-generic point. To simplify the exposition we will assume by way of contradiction that $\operatorname{dim}_{\mathcal{M}} N=n>1$.

Very much like in the 1-dimensional case, the proof goes through finding an $\mathcal{M}$-definable set $X$, and an $\mathcal{N}$-definable (partial quasi) order $\leq_{t}$ such that the restriction of $\leq_{t}$ to $X$ is linear. We start with some definitions and results preparing the ground for what follows. We keep the notation of the previous section.

Definition 3.2. Let $\mathcal{M}$ be an o-minimal structure and $S \subseteq X \subseteq M^{k}$ definable subsets, $X$ definable over $\emptyset . S$ is generically closed in $X$ if for every $b \in X$ generic over $\emptyset$, if $b \in \operatorname{cl}_{X}(S)$, then $b \in S$.

Note that the above definition is meaningful only for sets $S$ which are not $\emptyset$ definable. Throughout this section, by "S is generically closed" we will mean that $S$ is generically closed in $N$.

Lemma 3.3. Suppose $\mathcal{N}:=\left(N, \leq_{p}\right)$ is an $\mathcal{M}$-definable structure such that $N$ is $\mathcal{N}$-minimal with $\operatorname{dim}_{\mathcal{M}} N=n$. Let $Z \subseteq N$ be any $\mathcal{N}$-definable set and $\leq_{t}$ the (partial) quasi order defined by

$$
a \leq_{t} b \Leftrightarrow\left|\left\{y \in Z \mid y \leq_{p} a \wedge y \not_{p} b\right\}\right|<\infty .
$$

Then for any $a \in N$ the set $\left\{x \mid x \leq_{t} a\right\}$ is generically closed in $N$.

Proof. The lemma is trivial if $Z$ is finite, so we assume it not to be the case. Notice that in order to prove the lemma we must show first that $\leq_{t}$ is in fact a quasi order and second that the cones it defines are generically closed. 
To prove it is a quasi order, let $a, b, c$ be elements in $N$ such that $a \leq_{t}$ $b \leq_{t} c$. Setting $L(x):=\left\{y \mid y \leq_{p} x\right\}$ we know that both $(L(b) \backslash L(a)) \cap Z$ and $(L(c) \backslash L(b)) \cap Z$ are finite.

But

$$
L(c) \backslash L(a) \subset(L(c) \backslash L(b)) \cup(L(b) \backslash L(a))
$$

so $(L(c) \backslash L(a)) \cap Z$ is finite and by definition $a \leq_{t} c$ so $\leq_{t}$ is transitive.

To prove that $L_{t}(a)$ is generically closed, suppose that $c \not Z_{t} a$ for some $c$ generic over $\emptyset$. By definition there are infinitely many points in $Z \cap(L(c) \backslash$ $L(a))$. Since this last set is $\mathcal{N}$-definable we know by assumption that it has dimension $n$ so there is an open set $U \subseteq Z \cap L(c)$ such that $U \cap L(a)=\emptyset$. Choosing $U$ small enough, we may assume that it is definable over parameters independent over all the data, so by genericity of $c$ there is a neighbourhood $V$ of $c$ such that $U \subseteq L(x) \cap Z$ for all $x \in V$ whence $x \not Z_{t} a$ as required.

It will be important for applications to note that in the above lemma we do not assume that $Z$ is $\emptyset$-definable.

Out next step is to find an $\mathcal{N}$-definable order $\leq_{t}$ and an $\mathcal{M}$-definable line $l$ through $\mathcal{N}$ (see below) such that $\leq_{t}$ restricted to $l$ is a linear order. Our way of obtaining this is reminiscent of Shelah's proof of Theorem 1.7 .

The following technical result will be needed:

Proposition 3.4. Let $\mathcal{N}:=\left(N, \leq_{p}\right)$ be a partially ordered set with infinite chains definable in an o-minimal structure $\mathcal{M}$ and $\operatorname{dim}_{\mathcal{M}} N=n$. Assume also that $N$ is $\mathcal{N}$-minimal. Then:

(1) There is an infinite $\leq_{p}$-chain $a_{0} \leq_{p} \ldots \leq_{p} a_{i} \leq_{p} a_{i+1} \leq_{p} \ldots$ of elements in $N$ such that $a_{i}$ is generic for all $i$.

(2) There is an $\mathcal{M}$-generic type $p(x) \in S_{1}(\emptyset)$ and an infinite $\leq_{p^{-}}$chain $a_{0} \leq_{p} \ldots \leq_{p} a_{i} \leq_{p} a_{i+1} \leq_{p} \ldots$ such that $\models p\left(a_{i}\right)$ for all $i$.

(3) There is a type $p(x) \in S_{1}(\emptyset)$ and elements $a, b \models p$ such that $b$ is a generic element in $\partial G(a)$.

Proof.

(1). By assumption we have an infinite $\leq_{p}$-chain so by compactness we can find a sequence $\left\langle x_{(i, j)}\right\rangle_{(i, j) \in \omega \times \omega}$ where $x_{\left(i_{1}, j_{1}\right)} \leq_{p} x_{\left(i_{2}, j_{2}\right)}$ if and only if $\left(i_{1}, j_{1}\right)<\left(i_{2}, j_{2}\right)$ in the lexicographic order. Let $c_{i}:=x_{(i, 0)}$; by definition $\left(c_{i}, c_{i+1}\right)_{\leq_{p}}$ is infinite for any $i \in \omega$.

By $\mathcal{N}$-minimality $\operatorname{dim}\left(c_{i}, c_{i+1}\right)_{\leq_{p}}=n$ so there is some $\mathcal{M}$-generic $a_{i} \in$ $\left(c_{i}, c_{i+1}\right)_{\leq_{p}}$. Then $\left\langle a_{i}\right\rangle$ is an infinite $\leq_{p}$-chain of $\mathcal{M}$-generic elements.

(2). Using (1) and compactness we can find an arbitrarily long $\leq_{p}$-chain of $\mathcal{M}$-generic elements. By Ramsey's Theorem we can find a $\leq_{p}$-chain $\left\langle a_{i}\right\rangle$ of $\mathcal{M}$-generic elements such that $\operatorname{tp}\left(a_{i} / \emptyset\right)=\operatorname{tp}\left(a_{j} / \emptyset\right)$ for all $i, j$.

(3). By (2) there is an $\mathcal{M}$-generic type $p(x)$ such that there are infinite $\leq_{p}$-chains among realizations of $p(x)$. Let $a \models p(x)$ so that for any $\phi(x) \in$ $p(x)$ both $G(a) \cap \phi(N)$ and $\neg G(a) \cap \phi(N)$ have dimension $n$. By [Joh01] 
$\operatorname{dim} \partial G(a) \cap \phi(N)=n-1$ for any $\phi(x) \in p(x)$. By compactness there exists $b \in \partial G(a) \cap p$ such that $\operatorname{dim}(b / a)=n-1$. The elements $a, b$ will satisfy the requirements of $(3)$.

A line through $N$ is a 1 -dimensional ( $\mathcal{M}$-definable) definably connected subset of $N$. Say that a line $l$ through $N$ is generic if any generic $a \in l$ is generic also (over $\emptyset$ ) in $N$. So a line $l$ through $N$ is definably homeomorphic to an interval in $\mathcal{M}$. Fixing such a homeomorphism, $l$ inherits an ordering form $\mathcal{M}$. Throughout this section, we will assume implicitly that lines come equipped with some such ordering. The only requirement we will make is that when working with a family of lines the ordering on all lines is given uniformly.

Let $l$ be a line through $N$. Given $b \in X$ there are unique maximal closed intervals $I_{0}, I_{1}, \ldots, I_{n}$ such that

(1) The right endpoint of $I_{j}$ is the left endpoint of $I_{j+1}$.

(2) $l=\bigcup_{i} I_{i}$

(3) Either $I_{j}=\overline{I_{j} \cap G(b)}$ or $I_{j}=\overline{I_{j} \backslash G(b)}$.

Whenever these conditions hold we will say that $\bigcup_{i} I_{i}$ (or $\bigcup_{i} I_{i}(b)$ if we want to make $b$ explicit) is the partition of $G(b) \cap l$ (or the partition of $l$ with respect to $b$ ).

If $\bigcup_{i=1}^{r} I_{i}$ is a partition of $G(b) \cap l$ for fixed $l$ and $b$ let $f_{l}^{b}: r \rightarrow 2$ be such that $f_{l}^{b}(i)=1$ if $I_{j}=\overline{I_{j} \cap G(b)}$ and $f_{l}^{b}(i)=0$ otherwise; let

$$
s_{l}(b):=\left\langle f_{l}^{b}(i)\right\rangle .
$$

We will say that $s_{l}(b)$ has a sign change at $j$ if $f(j)=0$ and $f(j+1)=1$ or vice versa.

For a fixed line $l$ say that $\bigcup_{i} I_{i}$ is locally constant at $b$ if for every open neighbourhood $U$ of $b$ the set

$$
\left\{y \mid \bigcup_{i} I_{i} \text { is a partition of } G(y) \cup I\right\}
$$

contains an open subset of $U$. We will say that $\bigcup_{i} I_{i}$ is locally constant if it is locally constant at some $b \in X$.

Finally, given any family $\mathcal{B}$ of pairs $(l, b)$ as above, we will say that the sequence $s_{l}(b)$ (originating from the partition of $l$ with respect to $b$ ) is maximal in $\mathcal{B}$ if it has a maximum number of sign changes (i.e. $\left|s_{l}(b)\right|$ is maximal) among all $s_{l^{\prime}}\left(b^{\prime}\right)$ with $\left(l^{\prime}, b^{\prime}\right) \in \mathcal{B}$; we will call $s_{l}(b)$ the type of the partition $G(b) \cap l$.

Claim 3.5. Fix a line $l$. Then $l$ admits only finitely many locally constant partitions.

Proof. Let $A$ be any set such that $l$ is definable over $A$. Suppose that $\bigcup_{i} I_{i}$ is a locally constant partition, and let $a_{1}, \ldots, a_{r}$ be the left endpoints of $I_{1}, \ldots, I_{r}$ respectively. Let $U$ be the set of points such that $\bigcup_{i} I_{i}$ is a partition of $G(b) \cap I$ 
for all $b \in U$. By hypothesis $U$ has dimension $n$ so there is some $b \in U$ generic over $A \cup\left\{a_{1}, \ldots, a_{r}\right\}$. Clearly $a_{i} \in \operatorname{dcl}(b, A)$ and $b \downarrow_{A} a_{1}, \ldots, a_{r}$ so by symmetry $a_{i} \in \operatorname{dcl}(A)$. But the set of $x \in l$ such that $x$ is an endpoint of an interval in a constant partition of $l$ is $\mathcal{M}$-definable. Since it is contained in $\operatorname{acl}(A)$ it must be finite.

It is not hard to verify that Proposition 3.4 implies the existence of a generic line through $N$ admitting a non locally constant partition. Formally, (3) of the proposition implies that we can find $a \in N$ generic and $b \in \partial G(a)$ generic as such, such that $b / \emptyset$ is generic as well. This implies, in particular, that $\operatorname{dim}\left\{a^{\prime} \mid b \in \partial G\left(a^{\prime}\right)\right\}<n$. Since $a$ was generic, any line $l$ through $b$ will have non-constant partitions (witnessed by $a$ ) and, since $b / \emptyset$ is generic, if $l$ is chosen so that $b$ is generic on $l$ we get that this line is generic. Observe, moreover, that for such $a$ there is a neighbourhood $U$ of $a$ such that for no $a^{\prime} \in U$ is the partition $a^{\prime}$ induces on $l$ locally constant. So the set of $a^{\prime}$ inducing a non-constant partition on $l$ is $n$-dimensional.

Fix such $l_{0}$ and $b_{0}$ and let $L$ be a definable family of lines through $N$ of which $l_{0}$ is a generic member. Let $\mathcal{B}$ be the subset of $N \times L$ of all $\left(b^{\prime}, l^{\prime}\right)$ such that there exists an open set $V$ containing $b^{\prime}$ where $G(y) \cap l^{\prime}$ is a non constant partition of $l^{\prime}$ of the same type as $G\left(b^{\prime}\right) \cap l^{\prime}$ for all $y \in V$. Let $l \in L$ be generic and $(b, l) \in \mathcal{B}$ be such that the partition of $l$ with respect to $b$ is maximal among all elements in the set

$$
\left\{b^{\prime} \mid\left(b^{\prime}, l\right) \in \mathcal{B}\right\} .
$$

By definition, for all $b^{\prime} \in M$ if the size of the partition $G\left(b^{\prime}\right) \cap l$ is greater than the size of the partition of $G(b) \cap l$ then either $G\left(b^{\prime}\right) \cap l$ is a constant partition or $b^{\prime}$ is not generic (over $l$ ). Specifically,

\section{(a) $\quad \operatorname{dim}\left\{b^{\prime} \mid G\left(b^{\prime}\right) \cap l\right.$ non constant and $\left.\left|s_{l}\left(b^{\prime}\right)\right|>\left|s_{l}(b)\right|\right\}<n$.}

From now on we fix $b, l$ as above.

Remark 3.6. Let $a$ be a generic point of $l$. If $a \in G\left(b^{\prime}\right)$ is isolated in $G\left(b^{\prime}\right) \cap l$ then (by symmetry) $\operatorname{dim}\left(b^{\prime} / a\right)<n$.

Lemma 3.7. Let $\mathbb{D}$ be the set of all $d \in N$ such that $\left|s_{l}(d)\right|>\left|s_{l}(b)\right|$. Then there is an $\mathcal{N}$-definable set $\mathbb{D}^{\prime}$ such that $\operatorname{dim} \mathbb{D}^{\prime} \triangle \mathbb{D}<n$. In particular, any infinite $\mathcal{N}$-definable subset of $N \backslash \mathbb{D}^{\prime}$ intersects $N \backslash \mathbb{D}$ in an infinite set.

Proof. By Claim 3.5 we can find $d_{1}, \ldots, d_{k}$, representatives of the locally constant partitions of $l$, such that for any $y$ if the partition of $G(y) \cap l$ is locally constant and $\left|s_{l}(y)\right|>\left|s_{l}(b)\right|$ then $G(y) \cap l=G\left(d_{i}\right) \cap l$ for some $i$.

Let $J_{0}$ be the set of $(i, j)$ such that $f_{l}^{d_{j}}(i)=0$ and let $J_{1}$ be the set of $(i, j)$ such that $f_{l}^{d_{j}}(i)=1$. Let $c_{i j} \in I_{i}\left(d_{j}\right)$ be generic points in $l$ for all $(i, j)$. 
Let

$$
D_{j}:=\left\{x \mid \bigwedge_{(i, j) \in J_{1}} c_{i j} \in G(x) \wedge \bigwedge_{(i, j) \in J_{0}} c_{i j} \notin G(x)\right\} .
$$

If $x \in D_{j}$ then either $\left|s_{l}(b)\right|<\left|s_{l}(x)\right|$, implying that $x \in \mathbb{D}$, or $c_{i j}$ is an isolated point in $G(x) \cap l$ for some $i \in J_{0}$. By remark 3.6 this implies that the latter case can only occur if $\operatorname{dim} x / l<n$. Conversely, if $\left|s_{l}(x)\right|>\left|s_{l}(b)\right|$ then either $x$ induces on $l$ a constant partition, in which case $G(x) \cap l=G\left(d_{j}\right) \cap l$ for some $j$ and $x \in D_{j}$; or $x$ is not generic in $\{x \mid(x, l) \in \mathcal{B}\}$ by (h) above. Setting

$$
\mathbb{D}^{\prime}:=\bigcup_{j} D_{j}
$$

the conclusion of the lemma follows.

Recall that we fixed some generic $b$ inducing a partition of $l$ maximal among all generic $b^{\prime}$ such that $\left(b^{\prime}, l\right) \in \mathcal{B}$. Let $J_{1}:=\left\{j \mid f_{l}^{b}(j)=1\right\}$ and $J_{0}:=\left\{j \mid f_{l}^{b}(j)=0\right\}$. Let $a_{j}$ be generic points in the interior of $I_{j}(b)$. Define:

$$
Z_{0}:=\left\{y \mid \bigwedge_{j \in J_{1}} a_{j} \in G(y) \wedge \bigwedge_{j \in J_{0}} a_{j} \notin G(y)\right\}
$$

and

$$
Z:=\left\{y \in Z_{0} \mid y \notin \mathbb{D}^{\prime}\right\} .
$$

Finally, we can define our partial order $\leq_{t}$ :

$$
x_{1} \leq_{t} x_{2} \Longleftrightarrow\left|\left\{y \in Z \mid y \leq_{p} x_{1} \wedge y \not_{p} x_{2}\right\}\right|<\infty .
$$

By Lemma $3.3 \leq_{t}$ is indeed a partial order, and $\left\{y \mid y \leq_{t} a\right\}$ is generically closed for all generic $a \in N$. We now show that $\leq_{t}$ has the property we were looking for, namely that restricted to some line (not surprisingly, $l$ ) through $N$ it is linear.

To see this note that, as $G(b)$ does not induce a constant partition on $l$, there is some endpoint $d$ of one of the intervals in $G(b) \cap l$ witnessing it. Because $d$ is generic in $l$ we can find a neighbourhood $V \cap l$ of $d$ such that (without loss of generality) for all $d_{1}<d_{2}\left(d_{1}, d_{2} \in V \cap l\right)$ if for some $y$ we have

$$
\bigwedge_{j \in J_{1}} a_{j} \in G(y) \wedge \bigwedge_{j \in J_{0}} a_{j} \notin G(y) \wedge d_{1} \in G(y) \wedge d_{2} \notin G(y)
$$

then either $\left|s_{l}(y)\right|>\left|s_{l}(b)\right|$ and $y \in \mathbb{D}$ or $a_{j}$ is an isolated point in $G(y)$ for some $j \in J_{1}$. Since $\operatorname{dim}\left(\mathbb{D} \triangle \mathbb{D}^{\prime}\right)<n$ by Remark 3.6 there can be only finitely many $y$ outside $\mathbb{D}^{\prime}$ satisfying $(*)$. So restricted to $V \cap l, \leq_{t}$ is a quasi order agreeing with the natural order on $l$.

It remains to verify that $\leq_{t}$ is an order (on $V \cap L$ ). But since $b$ is generic over all the data there are neighbourhoods $U$ of $b$ and $W \subseteq l$ of $d$ such that 
for all $d^{\prime} \in W$ there is some $b^{\prime} \in U$ with $d^{\prime}$ an endpoint of an interval in the partition of $l$ induced by $b^{\prime}$ and $a_{i} \in G\left(b^{\prime}\right)$ if and only if $a_{i} \in G(b)$ (see Figure 1 below). So for every $x_{1} \neq x_{2} \in W$ there are infinitely many $b^{\prime}$ in $U$ separating them and $x_{1} \not_{t} x_{2}$.

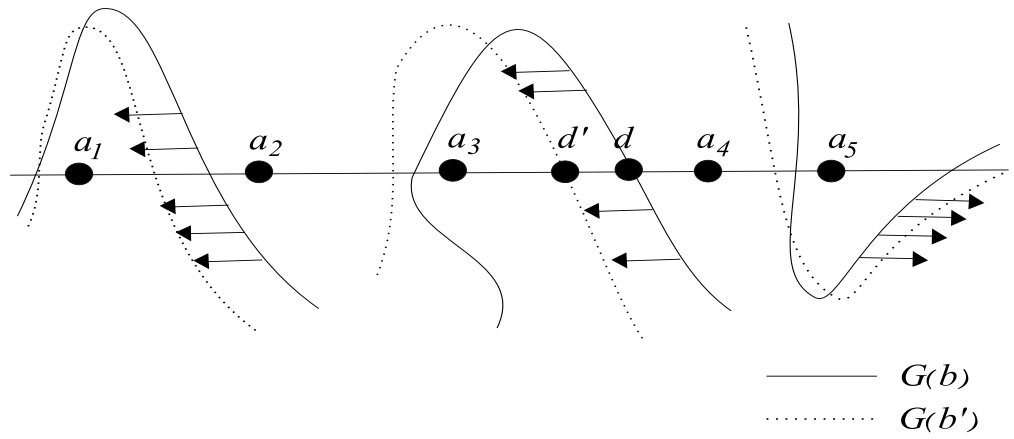

Figure 1: $d^{\prime} \leq_{t} d$

3.1. Going down. We can now prove the main result of this section:

Theorem 3.8. Let $\mathcal{N}$ be definable (over $\emptyset$ ) in an o-minimal structure $\mathcal{M}$. Assume that $\operatorname{dim}_{\mathcal{M}} N=n>1$. Then there exists an infinite $\mathcal{N}$-definable (over $B$ ) set $X \subseteq N$ such that $\operatorname{dim}(X)<n$ and $U(\operatorname{tp}(g / \emptyset))=\infty$ for some $g \in\left(X \backslash \operatorname{acl}_{\mathcal{M}}(B)\right)$

Proof. Assume not. So we can construct an $\mathcal{N}$-definable (quasi) order $\leq_{t}$ linear on some generic line $l$ through $\mathcal{N}$. By Lemma 3.3 the lower cones of $\leq_{t}$ are generically closed. We fix $\leq_{t}, l$ and $a, b \in l$ such that $\leq_{t}$ is linear on $[a, b]_{\leq_{t}} \cap l$.

We need some notation and easy observations. First, by the genericity of $l$, any $(\mathcal{M})$-generic $c \in l$ is also $\mathcal{M}$-generic over $\emptyset$. Denote $[a, b]^{l}:=[a, b]_{\leq_{t}} \cap l$ and let

$$
m(y):=\max _{[a, b]^{l}}\left\{x \in(a, b)^{l} \mid x \leq_{t} y\right\}
$$

for any $y \in N$ ( $m$ need not be globally defined). With this notation, if $y \in(a, b)_{\leq_{t}}$ then $m(y) \in[a, b]^{l}$ and since $m(y) \in \operatorname{cl}\left\{x \in(a, b)^{l} \mid x \leq_{t} y\right\}$ Lemma 3.3 assures that $m(y) \leq_{t} y$ whenever $m(y)$ is generic in $l$.

Claim 3.9. We may assume that $\operatorname{dim}\left(m^{-1}(c)\right)<n$ and that $c \leq_{t} m^{-1}(c)$ for all $c \in(a, b)^{l}$.

Proof. Since $\operatorname{dim}(N)=n$, necessarily, $\operatorname{dim}\left(m^{-1}(c)\right)<n$ for all but finitely many $c$. On the other hand the formula $\exists y\left(m(y)=c \wedge c \not_{t} y\right)$ implies that $c$ is not generic in $l$ so there are only finitely many such.

Thus, by shrinking our interval if necessary, we can find some $\left[a^{\prime}, b^{\prime}\right]^{l}$ subinterval of $[a, b]^{l}$ such that $\operatorname{dim}\left(m^{-1}(c)\right)<n$ for all $c \in\left[a^{\prime}, b^{\prime}\right]^{l}$. Replacing $[a, b]^{l}$ with $\left[a^{\prime}, b^{\prime}\right]^{l}$ it is easy to verify that the claim follows.

Claim 3.10. If $\operatorname{dim}\left(m^{-1}(x)\right)<n-1$ for all $x \in(a, b)^{l}$ then $\operatorname{dim}\left((a, b)_{\leq_{t}}\right)<$ $n$. 
Proof. If $a<_{t} x<_{t} b$ then $a \leq m(x) \leq b$ so

$$
(a, b)_{\leq_{t}} \subseteq \bigcup_{x \in[a, b]^{l}} m^{-1}(x) .
$$

Therefore $\operatorname{dim}\left(m^{-1}(x)\right)<n-1$ for all such $x$ implies, by the additivity of o-minimal dimension, that $\operatorname{dim}\left((a, b)_{\leq_{t}}\right)<n$.

Because $(a, b)_{\leq_{t}}$ has infinite $\leq_{t}$-chains (e.g. the interval in $\left.(a, b)^{l}\right)$ it must be unstable; so if $\operatorname{dim}(a, b)_{t_{t}}<n$ the theorem follows. Hence we may assume that $\operatorname{dim}\left(\left(a^{\prime}, b^{\prime}\right)_{\leq_{t}}\right)=n$ for all $a<a^{\prime}<b^{\prime}<b$ in $l$ and that, by Claim 3.10, $\operatorname{dim}\left(m^{-1}(x)\right)=n-1$ for infinitely many $x \in(a, b)^{l}$. By o-minimality of $l$ (and definability of the o-minimal dimension) there is a subinterval $\left(a^{\prime}, b^{\prime}\right)^{l} \subseteq(a, b)^{l}$ such that $\operatorname{dim}\left(m^{-1}(x)\right)=n-1$ for all $x \in\left(a^{\prime}, b^{\prime}\right)^{l}$.

Claim 3.11. Let $a^{\prime}, b^{\prime} \in l$ be such that $\operatorname{dim}\left(m^{-1}(x)\right)=n-1$ for all $x \in$ $\left(a^{\prime}, b^{\prime}\right)^{l}$. Then $U(\operatorname{tp}(g / A))=\infty$ for any parameter set $A$ and $g \in\left(a^{\prime}, b^{\prime}\right)_{\leq_{t}}$ generic over $A$.

Proof. Fix $A$ and $g \in\left(a^{\prime}, b^{\prime}\right)_{\leq_{t}}$ generic over $A$. Let $\left\langle g_{i}\right\rangle_{i \in \mathbb{Q}}$ be an $\mathcal{M}$ independent $\mathcal{M}$-indiscernible sequence in $\operatorname{tp}_{\mathcal{M}}(g / A)$. Since $g$ is generic over $A$, in particular, $m(g)$ is generic in $l$, and therefore $m\left(g_{i}\right) \neq m\left(g_{j}\right)$ for all $i \neq j$. Let $c_{i}:=m\left(g_{i}\right)$ so the sequence $\left\langle c_{i}\right\rangle_{i \in \mathbb{Q}}$ is a $\leq_{t}$-linearly-ordered indiscernible sequence, so without loss of generality it is $\leq_{t}$-increasing.

By hypothesis $c_{i} \unlhd_{t} g_{j}$ if and only if $i<j$. As in the case of dense linear orders, the formula $\left(c_{i} \succ_{t} x\right) \wedge \neg\left(c_{j} \succ_{t} x\right)$ divides over $A$, and is realized by $g_{k}$ for all $i<k<j$. Since our set is indexed by $\mathbb{Q}$ we get an infinite dividing sequence which witnesses that $\mathrm{U}(\operatorname{tp}(g / A))=\infty$.

From now on, we will assume $a^{\prime}, b^{\prime} \in l$ are such that $\operatorname{dim}\left(m^{-1}(x)\right)=n-1$ and $m(x) \leq_{t} x$ for all $x \in\left(a^{\prime}, b^{\prime}\right)_{\leq_{t}}$. Let $g \in\left(a^{\prime}, b^{\prime}\right)_{\leq_{t}}$ be generic over all the parameters defining $l$, so by the last claim $\mathrm{U}(\operatorname{tp}(g / l))=\infty$. Denoting $c=m(g)$ we know that $c$ is generic in $\left(a^{\prime}, b^{\prime}\right)^{l}$. The following lemma will complete the proof of Theorem 3.8.

Lemma 3.12. Let $g, c$ be as above then there exists a set $X$ with $\operatorname{dim} X<n$ such that either $X$ is $\mathcal{N}$-definable over $c$ and $g \in X$ or $X$ contains an infinite $\leq_{t}$-chain

Proof. Since $g$ is generic over $l$ and $\operatorname{dim}(c / l)=1$ the additivity of o-minimal dimension gives $\operatorname{dim}(\operatorname{tp}(g / c))=n-1$. So any $c$-definable set containing $g$ is non algebraic.

Notice that by density of $\leq_{t}$ on $\left(a^{\prime}, b^{\prime}\right)^{l}$ if $y \in\left(a^{\prime}, b^{\prime}\right)^{l}$ is such that $c \neq m(y)$ then either $(c, y)_{\leq_{t}}$ is empty or it contains an infinite $\leq_{t}$-chain (and infinitely many points) in $(a, b)^{l}$. Writing

$$
X_{n}(c):=\left\{y \mid(c, y)_{\leq_{t}} \text { does not contain } \leq_{t} \text {-chains of size larger than } n\right\}
$$

this implies that either $g \in X_{n}(c)$ for some $n \in \mathbb{N}$, and $X_{n}(c)$ being $\leq_{t^{-}}$ definable the lemma follows, or $g \notin X_{n}(c)$ for all $n$. 
In the latter case, by saturation of $\mathcal{M}$, the interval $(c, g)_{\leq_{t}} \subseteq m^{-1}(c)$ contains infinite $\leq_{t}$-chains. By assumption, this implies that $\operatorname{dim}(c, g)_{t_{t}}<n$ and being unstable it satisfies the conclusion of the lemma.

This finishes the proof of the theorem.

3.2. An alternative proof. In this subsection we propose a different approach to the proof of Theorem [3.8. We find the proof instructive in the way it allows us to control local phenomena in reducts of o-minimal theories. However, being technically more involved, we do not give all the details. For simplicity and concreteness we discuss the case $N \subseteq M^{2}$ and $\operatorname{dim} N=2$. The interested reader should not find it hard to convince himself (or herself, or others) that the proof extends to the general case, but even the uninterested reader would probably not need much convincing in agreeing that the proof is unpleasant enough as it is, even without the additional technicalities such a generalisation would require.

To make life a little easier we will prove a slightly weaker version of the theorem. We prove:

Theorem 3.13. Let $\mathcal{N}$ be definable in an o-minimal structure $\mathcal{M}$. If $\mathcal{N}$ is unstable and $\operatorname{dim} N=n>1$ then there exists an infinite $\mathcal{N}$-definable $X \subseteq N$ such that $\operatorname{dim} X<n$.

The proof starts at the same point where Section 3.1 does, and we keep the assumptions and notation accumulated up to that point. In particular, we have a fixed generic line $l$ through $N$ and the $\mathcal{N}$-definable order $\leq_{t}$ obtained above. Recall that the restriction of $\leq_{t}$ to $l$ coincides with the natural, ominimal, order on $l$ (induced from some definable homeomorphism of $l$ with an interval in $M$ ). Because of the assumption that $N$ is of full dimension (in $M^{2}$ ) we may choose the line $l$ parallel to one of the axis. In the general case $\left(N \subseteq M^{k}\right.$ and $\left.\operatorname{dim} N=n\right)$ we choose an $n$-dimensional cell $N^{\prime} \subseteq N$ which is naturally definably homeomorphic to an open box $B \subseteq M^{n}$; in that case we choose $l^{\prime}$ through $B$ parallel to one of the axis and set $l$ to be the image of $l^{\prime} \cap B$ under the inverse homeomorphism.

The assumption that $\leq_{t}$ agrees with the order on $M$ on some line $l$ through $N$ implies that there is an infinite set of $x \in N$ such that $x$ is not an isolated point in $\operatorname{cl}\left(L_{t}(x)\right)$. The first part of the proof consists in showing that if for some $a$, generic over all the data (including the parameters required to define $\left.\leq_{t}\right), a$ is not isolated in $L_{t}(a)$ then the desired result follows. We will then show how to change $\leq_{t}$ to obtain such an $\mathcal{M}$-generic $a$.

For the first part we need the following easy claim:

Claim 3.14. Let $a \in N$ be $\mathcal{M}$-generic and $c \in \partial L_{t}(a)$ be generic as such. If, in addition, $c / \emptyset$ is generic then $\left\{z \mid c \leq_{p} z \leq_{p} a\right\}$ is finite and $\left\{b \in L_{t}(a) \mid\right.$ $\left.\left|(b, a)_{t}\right|<\infty\right\}$ is n-dimensional.

Proof. As we have already showed we may assume that $\leq_{t}$ is generically closed (i.e., that $L_{t}(x)$ is generically closed for all generic $x$ ). Assume that 
$a, c$ are as above and that $c / \emptyset$ is generic, therefore $c £^{\mathcal{M}} a$. Hence, $\operatorname{dim}\left\{a^{\prime} \mid\right.$ $\left.c \in \partial L_{t}\left(a^{\prime}\right)\right\}<n$ and $c \in L_{t}(a)$.

By construction, if $c \leq_{t} d \leq_{t} a$ then $c \in \partial L_{t}(d)$; so for any such $d$ we have $c \not^{\mathcal{M}} d$ which implies $\operatorname{dim}\left(\left\{z \mid c \leq_{t} z \leq_{t} a\right\}\right)<n$. But $\left\{z \mid c \leq_{t} z \leq_{t} a\right\}$ is $\mathcal{N}$-definable so by assumption it must be finite.

Since $b$ was generic in $\partial L_{t}(a)$ we get that $\left\{b \in L_{t}(a)||(b, a)_{\leq_{t}} \mid<\infty\right\}$ is infinite, so it must be $n$-dimensional.

On the other hand, if $a \in N$ is generic and $a$ is not isolated in $L_{t}(a)$ then for every $b \in L_{t}(a)$ generic over $a$ it must be that $a \in \operatorname{Int} G_{t}(b)$. Thus the $\leq_{t}$-interval $(b, a)_{\leq_{t}}$ is infinite, contradicting the previous claim.

We can now turn to showing that there is some partial order with infinite chains $\leq_{t}$ and a generic $a$ such that $a$ is not an isolated point in $L_{t}(a)$. As in the previous proof, we fix $b$ generic inducing a partition on $l$ maximal among all generic $b^{\prime}$ such that $\left(b^{\prime}, l\right) \in \mathcal{B}$. We also fix generic $a_{j} \in I_{j}(b)$. Denote $A$ the collection of the $a_{j}$ and

$$
R_{b}(x, A):=\left(\bigwedge_{a_{j} \in A}\left(a_{j} \in G(x) \Longleftrightarrow a_{j} \in G(b)\right)\right) .
$$

Say that $x$ is good for $A$ (with respect to $b$ ) if $\models R_{b}(x, A)$. Observe that, since $b$ was generic and the $a_{i}$ were chosen independent of $b$ (over all the data), if $x$ is good for $A$ with respect to $b$ it is good for $A$ with respect to any $b^{\prime}$ close enough to $b$.

For each $a_{i} \in A$ let $B_{i} \ni a$ be an open box such that $B_{i} \subseteq G(b)$ if $a_{i} \in G(b)$ and $B_{i} \cap G(b)=\emptyset$ otherwise. Let $l^{\prime}$ be a line parallel to $l$ such that $l^{\prime} \cap B_{i} \neq \emptyset$ for all $i$. Choosing $l^{\prime}$ close enough to $l$ we may assume that $G(b)$ induces a maximal non-constant partition of $l^{\prime}$ (as usual, among all generic $b^{\prime}$ such that $\left.\left(b^{\prime}, l\right) \in \mathcal{B}\right)$. Choose $a_{i}^{\prime} \in B_{i} \cap l^{\prime}$ witnessing this and denote this set of points $A^{\prime}$. It will be convenient to take $A^{\prime}$ to be the projection of $A$ to $l^{\prime}$.

We slightly change our definition of $\leq_{t}$. Instead of requiring that $x_{1} \leq_{t} x_{2}$ if and only if the set of $y \in Z$ such that $y \leq_{p} x_{1} \wedge y \mathbb{Z}_{p} x_{2}$ is finite (having $Z$ defined as above) we replace $Z$ by $Z^{\prime}$ where $Z^{\prime}:=\left\{z \in Z \mid \models R_{b^{\prime}}\left(z, A^{\prime}\right)\right\}$. I.e. we restrict ourselves to the set $Z^{\prime}$ of $b^{\prime}$ such that $G\left(b^{\prime}\right)$ induces a nonconstant maximal partition on both $l$ and $l^{\prime}$ and $b^{\prime}$ is good for both $l$ and $l^{\prime}$ (with respect to $b$ in both cases). Because $b$ is generic over all the data, $Z^{\prime}$ contains a small open neighbourhood of $b$ which must be a 2-dimensional set ( $n$-dimensional in the general case). Let $x \in \partial G(b)$ be generic over all the data (so in particular not on $l, l^{\prime}$ ) such that $x$ lies between $l$ and $l^{\prime}$ (see Figure 2). 


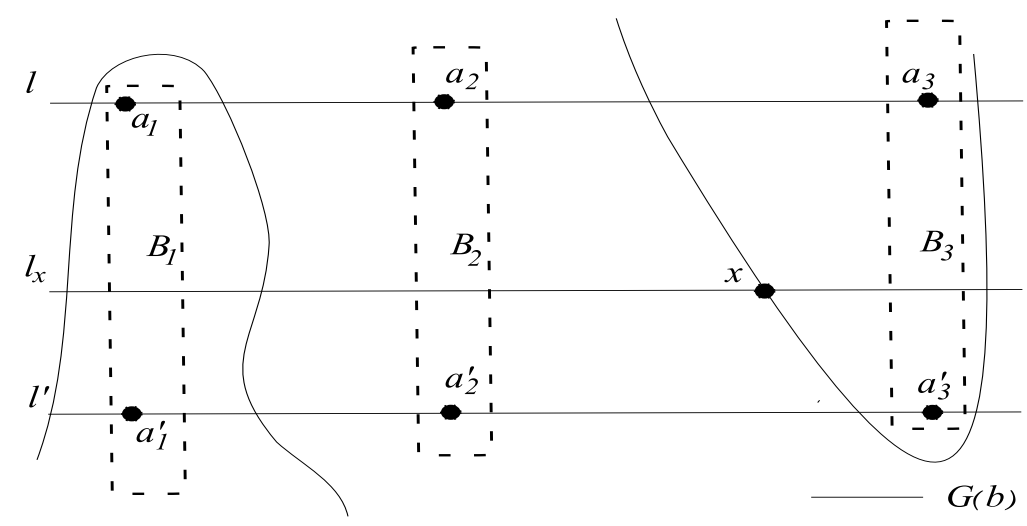

Figure 2

Let $l_{x}$ be the line through $x$ parallel to $l, l^{\prime}$. We would like to show that

$$
\left\{y \in l_{x} \mid x \leq_{t} y\right\} \text { has } x \text { in its boundary. }
$$

This will be enough ( $b$ was taken to be generic and $x$ is a generic point in $\partial G(b)$, so moving $b$ we will get a 2-dimensional set of $x$ with the required property). In the general $n$-dimensional case we would have to repeat the same process several times, but the main idea is unaltered.

We do not claim that this will be outright true, but we will now start a process which will provide the desired result. Throughout, when working within a line (parallel to one of the axis) we will use the natural induced order.

Assume $(* *)$ is not true. By definition $x \in \partial G(b)$ and for concreteness assume that for all $x<y$ in $l_{x}$ there is $z \in l_{x}$ with $x<z<y$ such that $z \in G(b)$ (as in the figure above). Since (**) is assumed not to hold, for all $y>x$ there exists some $z \in l_{x}$ between $x$ and $y$ such that $z \notin G_{t}(x)$. By the definition of $\leq_{t}$ this means that there are infinitely many $b^{\prime} \in Z^{\prime}$ such that $x \in G\left(b^{\prime}\right)$ but $z \notin G\left(b^{\prime}\right)$. Fix any such $\mathcal{M}$-generic $b^{\prime}$.

Let $\left(a_{i}, a_{i}^{\prime}\right)$ be the line segment between $a_{i}$ and $a_{i}^{\prime}$. Observe that if $a_{i} \in$ $G(b)$ then by choice of $l^{\prime}$ and $a_{i}^{\prime}$ the segment $\left(a_{i}, a_{i}^{\prime}\right)$ is contained in $B_{i}$ (and therefore in $G(b))$. Similarly, $\left(a_{i}, a_{i}^{\prime}\right) \cap G(b)=\emptyset$ if $a_{i} \notin G(b)$. Since $b^{\prime}$ was chosen generic over all the data, we also know that $G\left(b^{\prime}\right)$ contains open neighbourhoods of $\left(a_{i}, a_{i}^{\prime}\right)$ or open neighbourhoods disjoint from $G\left(b^{\prime}\right)$.

Assume first that for all $i$ we have $\left(a_{i}, a_{i}^{\prime}\right) \subseteq G\left(b^{\prime}\right)$ whenever $a_{i} \in G(b)$ and $\left(a_{i}, a_{i}^{\prime}\right) \cap G\left(b^{\prime}\right)=\emptyset$ otherwise. This implies that $l_{x}$ has more sign changes (witnessed by $G\left(b^{\prime}\right)$ ) than $l$ did (with respect to $b$ ) and it is easy to verify that $b^{\prime}$ (and $z$ ) can be chosen so that the partition is not constant. But $l$ was chosen so that the size of a maximal non-constant partition (with respect to a generic element $b$ ) is maximal, so we would have a contradiction.

So we may assume that for some $i$ either $a_{i} \in G(b)$ and $\left(a_{i}, a_{i}^{\prime}\right) \nsubseteq G\left(b^{\prime}\right)$ or $\left(a_{i}, a_{i}^{\prime}\right) \cap G\left(b^{\prime}\right) \neq \emptyset$ for some $a_{i} \notin G(b)$. For concreteness we will assume the former holds (see Figure 3) and let $a^{\prime \prime} \in\left(a_{i}, a_{i}^{\prime}\right)$ be such that $a_{i}^{\prime \prime} \notin G\left(b^{\prime}\right)$ be generic over all the data. 
We will now change our definition of $\leq_{t}$ once more replacing $Z^{\prime}$ with $Z^{\prime \prime}:=\left\{z \in Z^{\prime} \mid a^{\prime \prime} \notin G(z)\right\}$. Take $l^{\prime \prime}$ close enough to $l$ so that $G\left(b^{\prime}\right)$ induces on $l^{\prime \prime}$ a maximal non-constant partition. Now restart the whole process with $l, l^{\prime \prime}, G\left(b^{\prime}\right)$ and $Z^{\prime \prime}$. By o-minimality, this process of restricting $Z$ to obtain more intervals in $G(b) \cap\left(a_{i}, a_{i}^{\prime}\right)$ cannot go on forever. So after finitely many such changes we are reduced to the case where $\left(a_{i}, a_{i}^{\prime}\right) \subseteq G\left(b^{\prime}\right)$ if $a_{i} \in G(b)$ and $\left(a_{i}, a_{i}^{\prime}\right) \cap G\left(b^{\prime}\right)=\emptyset$ otherwise, which we already proved contradicted the fact that $x$ was a non isolated point in $L_{t}(x) \cap l_{x}$. This concludes the proof of the theorem.

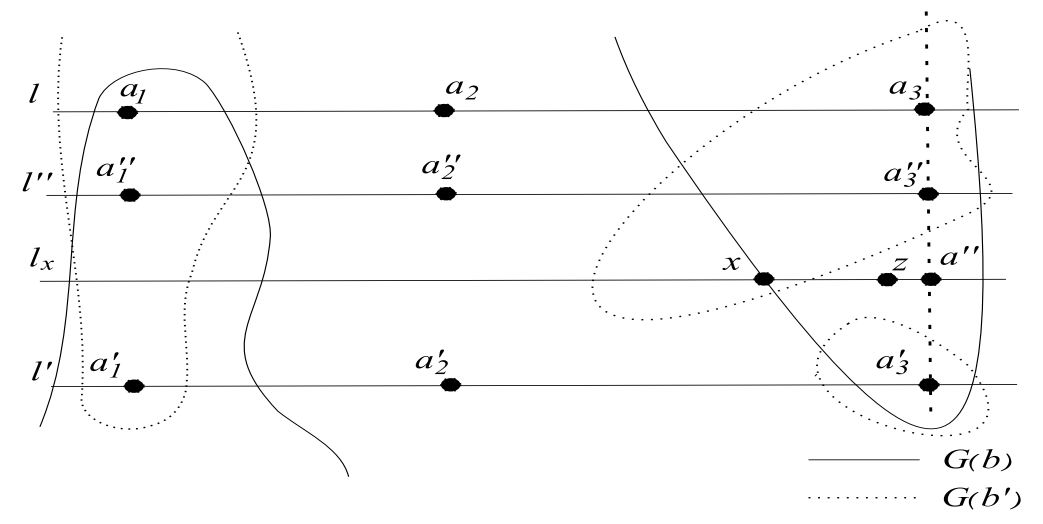

Figure 3

The results obtained thus far imply by induction that we can define a finite by o-minimal set in any purely unstable structure (see definition 4.17) interpretable in an o-minimal theory, proving the first part of Theorem 1.2, This theorem cannot be outright strengthened as the following example demonstrates. Consider the structure $\mathcal{N}:=\left(\mathbb{R}^{2}, \prec\right)$ where $\left(x_{1}, x_{2}\right) \prec\left(y_{1}, y_{2}\right)$ is interpreted as $x_{1}<y_{1}$. So $\mathcal{N}$ is unstable of o-minimal dimension 2 . It is an easy exercise to check that any infinite definable subset of $N$ is either 2-dimensional or stable, and that the only way to obtain an o-minimal structure in $\mathcal{N}$ is to work in $\mathcal{N}^{e q}$.

The above example is not the only obstacle on the way of completing the proof of Theorem 1.4. Towards that end we will also need to improve the results of this section in order to find an unstable $\mathcal{N}$-definable set of small $\mathcal{M}$-dimension. Before proceeding to this task, we conclude the present section with a discussion of the second part of Theorem 1.2.

Corollary 3.15. Let $\mathcal{N}$ be interpretable in an o-minimal $\kappa$-saturated structure $\mathcal{M}$ and $p \in S^{\mathcal{N}}\left(N_{0}\right)$ for some $N_{0} \prec N$ with $\left|N_{0}\right|<\kappa$. Then there exists a non-algebraic $p \subseteq q \in S^{\mathcal{N}}(N)$ which is either strongly stable or finite by o-minimal.

Since this result will not be used below we only give a sketch of the proof. If $p$ is strongly stable every non-algebraic $q \supseteq p$ is strongly stable and we have nothing to do. So we may assume that $p$ is weakly unstable. by extending $N_{0}$ if needed (preserving the cardinality) we may assume that 
$p$ is unstable, and by saturation we may also assume that there is an $\mathcal{N}$ definable partial order $\leq_{p}$ with infinite chains in $p$. If $\operatorname{dim}_{\mathcal{M}} p=1$ the result follows from Theorem 2.1. So we may assume that $\operatorname{dim}_{\mathcal{M}} p=n>1$. To simplify things we will assume that $\leq_{p}$ is $\emptyset$-definable in $\mathcal{N}$. For every $\phi \in p$ denote $N_{\phi}:=\{a \mid a \models \phi(x)\}$ and $\mathcal{N}_{\phi}$ the $\leq_{p}$-structure whose universe is $N_{\phi}$ and where $\leq_{p}$ is interpreted as its restriction therein. Clearly, $\mathcal{N}_{\phi}$ is unstable.

Our goal is to show that there exists a $\leq_{p}$-formula $\psi(x, y)$ over $\emptyset$ such that for all $\phi \in p$ there exists $c \subseteq N_{\phi}$ satisfying $0<\operatorname{dim}(\psi(x, c) \wedge \phi(x))<n$. By compactness (and the definability of o-minimal dimension) this would imply the existence of some $c$ such that $0<\operatorname{dim}(\psi(x, c) \wedge \phi(x))<n$ for all $\phi \in p$. In particular, we may assume that $0<\operatorname{dim} \psi(x, c)<n$. By assumption $\psi(x, c) \cup p$ is infinite, with the desired conclusion following by induction.

The starting point of the proof is the observation that, assuming Lemma 3.3 and Proposition 3.4, the proof of Theorem 3.8 assures that there are $a, b$ such that $0<\operatorname{dim}(a, b)_{\leq_{t}}<n$. Note also that the formula defining $\leq_{t}$ (given in $(\dagger)$ above) depends on $\mathcal{N}$ only by the use of parameters and, more significantly for us, by determining the maximal possible size of a nonconstant partition of a line through $\mathcal{N}$. We leave it as an exercise to verify that by saturation of $\mathcal{M}$, working in an infinitesimal (with respect to $N_{0}$ ) neighbourhood of some (generic) $e \models p$ we can find a line $l$ so small that the maximal size of a partition of $l$ in $\mathcal{N}_{\phi}$ is uniformly bounded (i.e. does not depend on $\phi$ ). Thus, under the assumption that Lemma 3.3 and Proposition 3.4 hold in any $\mathcal{N}_{\phi}$, the plan described in the previous paragraph can be carried out.

So we only have to take care of the case that one of the above propositions does not hold in cofinitely many of the $\mathcal{N}_{\phi}$. If Proposition 3.4 is the one that fails then, inspecting its proof, we get that there are $a, b$ such that $0<\operatorname{dim}\left((a, b)_{\leq_{p}} \cap \phi(\mathcal{M})\right)<n$ with the desired conclusion. If Lemma 3.4 is the one causing problems we need to note that the lemma is only used to show that $\leq_{t}$ (which, as we have observed, can be defined uniformly) is generically closed. If the proof of that statement fails, there must be some infinite set of the form $Z \cap(L(c) \backslash L(a)) \cap N_{\phi}$ which is of small dimension, for the definable set $Z$ appearing in $(\dagger)$. Since $Z$ does not depend on $\phi$ the conclusion follows.

\section{INTERPRETING AN O-MINIMAL STRUCTURE}

In this section we complete the induction introduced in the previous section to conclude that any unstable theory interpretable in an o-minimal structure interprets itself an o-minimal structure. As the example preceding Corollary 3.15 illustrates, in order to achieve this we cannot avoid working in $\mathcal{N}^{e q}$. Although it seems plausible that our argument could be carried out to $\mathcal{M}^{e q}$ this would require some additional technical tools. In order to 
avoid such technicalities we will assume from now on that $\mathcal{M}$ eliminates imaginaries.

We will work with both stable formulas and definable stable sets. To prevent any confusion we will use "stable" for definable stable sets and refer to stable formulas as formulas satisfying NOP.

4.1. Preliminaries. We need the some definitions and results from [Ons06] and $\mathrm{Ons} 02$.

Definition 4.1. A formula $\delta(x, a)$ strongly divides over $A$ if $\operatorname{tp}(a / A)$ is nonalgebraic and $\left\{\delta\left(x, a^{\prime}\right)\right\}_{a^{\prime} \models \operatorname{tp}(a / A)}$ is $k$-inconsistent for some $k \in \mathbb{N} . \quad \delta(x, a)$ $\mathrm{p}$-divides over $A$ if we can find some tuple $c$ such that $\delta(x, a)$ strongly divides over Ac.

Standard forking terminology generalises naturally to p-forking. For example, a formula $\mathrm{p}$-forks over a set $A$ if it implies a finite disjunction of formulas $\mathrm{p}$-dividing over $A$. In particular, $\mathrm{U}^{\mathrm{b}}$-rank is the foundation rank of the partial order (defined on complete types) $p<_{\mathrm{p}} q$ defines as " $p$ is a p-forking extension of $q$ ".

The p-rank of a formula is the analogue of the global rank in simple theories. That is, $\mathrm{p}(\varphi(x, b)) \geq \alpha+1$ if there is $\psi(x, c) \vdash \varphi(x, b) \mathrm{p}$-dividing over $b$ with $\mathrm{p}(\psi(x, c)) \geq \alpha$.

Fact 4.2. Let $\mathcal{N}$ be definable in an o-minimal structure $\mathcal{M}$, let $\phi(x, b)$ be $\mathcal{N}$-definable and let $p(x) \in S_{n}^{\mathcal{N}}(N)$. Then both $p(\phi(x, b))$ and $U^{b}(p(x))$ are finite.

Proof. Any instance of $\mathrm{b}$-forking in $\mathcal{N}$ implies an instance of $\mathrm{p}$-forking in $\mathcal{M}$ so the p-rank of any $\mathcal{N}$-definable set will be bounded by the dimension of the corresponding set in $\mathcal{M}$. This gives a finite bound for the global p-rank for structures interpretable in o-minimal theories which depends only on the arity of the variable $x$.

As in superstable theories (see [Pil96]), if we define

$$
\mathrm{p}(p(x)):=\min \{\mathrm{p}(\phi(x, b)) \mid \phi(x, b) \in p(x)\}
$$

then

$$
\mathrm{U}^{\mathrm{b}}(p(x)) \leq \mathrm{p}(p(x))
$$

which completes the proof.

The following is Theorem 5.1.1 in [Ons06]:

Fact 4.3. If $\phi(x, y)$ satisfies NOP and there is a $\phi$-formula witnessing that $\operatorname{tp}(a / b c)$ forks over $c$, then there is a $\phi$-formula witnessing that $\operatorname{tp}(a / b c)$ p-forks over $c$.

and

Fact 4.4. If $T$ is dependent and $\phi(x, b)$ is a definable stable set then for every formula $\psi(x, y)$ the formula $\phi(x, b) \wedge \psi(x, y)$ satisfies $N O P$. 
is well known, see for example [OP]. Gathering all of the above, we obtain:

Corollary 4.5. In a dependent theory, if a type $p$ contains a formula defining a stable set then $U^{b}(p)=U(p)$. In particular, if $U^{p}(p)$ is finite then $p$ has finite U-rank.

Proof. Any instance of $\mathrm{p}$-forking is an instance of forking so

$$
\mathrm{U}(p) \geq \mathrm{U}^{\mathrm{b}}(p)
$$

for any type $p$. We prove the other inequality by induction. For $\alpha=0$, $\mathrm{U}(p) \geq 0$ if and only if $p$ is consistent if and only if $\mathrm{U}^{\mathrm{b}}(p) \geq 0$. If $\mathrm{U}(p) \geq \alpha$ for $\alpha$ limit, the claim follows from the induction hypothesis and the fact that both $\mathrm{U}^{\mathrm{b}}(p) \geq \alpha$ and $\mathrm{U}(p) \geq \alpha$ if and only if $\mathrm{U}^{\mathrm{b}}(p) \geq \delta$ (resp. $\mathrm{U}(p) \geq \delta$ ) for all $\delta<\alpha$.

It remains to deal with $\mathrm{U}(p) \geq \alpha+1$. Assume inductively that for any type $q$ containing a formula defining a stable set, if $\mathrm{U}(q) \geq \alpha$ then $\mathrm{U}^{\mathrm{b}}(q) \geq \alpha$. Now let $p \in S(A)$ contain a formula $\phi(x, a)$ defining a stable set, and assume that $\mathrm{U}(p) \geq \alpha+1$.

By definition there is some $r \supseteq p$ such that $\mathrm{U}(r) \geq \alpha$ and $r$ forks over $A$ witnessed by some formula $\theta(x, b)$. Let $\theta^{\prime}(x, y):=\theta(x, y) \wedge \phi(x, a) ; \theta^{\prime}(x, y)$ satisfies NOP by Fact 4.4 and clearly $\theta^{\prime}(x, b)$ forks over $A$. By Fact 4.3 there is a $\theta^{\prime}$-formula which witnesses that $r$ p-forks over $A$, by induction $\mathrm{U}^{\mathrm{b}}(r) \geq \alpha$, and by definition $\mathrm{U}^{\mathrm{b}}(p) \geq \alpha+1$.

Fact 4.6. Let $\phi\left(x_{1}, x_{2}\right)$ be such that $\models \forall x_{1} \exists \leq n x_{2} \phi\left(x_{1}, x_{2}\right)$ for some $n \in \mathbb{N}$, and let $\phi^{\prime}(x):=\exists y \phi(x, y)$. Then the following hold.

- $\phi\left(\mathcal{C}^{2}\right)$ is a stable definable set if and only if $\phi^{\prime}(C)$ is a stable definable set.

- $p\left(\phi\left(\mathcal{C}^{2}\right)\right)=p\left(\phi^{\prime}(C)\right)$.

Proof. If $\phi^{\prime}$ is unstable, then any formula witnessing NOP for $\phi^{\prime}$ will also witness NOP for $\phi$. Thus, it's enough to show that if $\phi$ is unstable so is $\phi^{\prime}$. By 1.7 there is an indiscernible sequence $\overline{\mathbf{b}}$ satisfying $\phi$ and $\pi\left(x_{1}, y_{1} ; x_{2}, y_{2}\right)$ witnessing the strict order property. Let $b_{i}:=\left(a_{i}, c_{i}\right)$ be the i-th element of $\overline{\mathbf{b}}$; by assumption $c_{i} \in \operatorname{acl}\left(a_{i}\right)$. Therefore, $\operatorname{tp}\left(c_{1}, c_{2} / a_{1}, a_{2}\right)$ is isolated, say by $\psi\left(y_{1}, y_{2}, a_{1}, a_{2}\right)$. Hence, the formula $\left(\forall x_{1}, x_{2}\right)\left(\psi\left(y_{1}, y_{2}, x_{1}, x_{2}\right) \rightarrow\right.$ $\left.\pi\left(x_{1}, y_{1} ; x_{2}, y_{2}\right)\right)$ orders the sequence $\overline{\mathbf{a}}:=\left\{a_{i}\right\}_{i}$. This proves (i).

The proof of (ii) is a straightforward induction on the p-rank of $\phi^{\prime}(\mathcal{C})$.

Proposition 4.7. Let $\delta(x, y)$ and $\pi(y)$ be such that $\models \forall y\left(\pi(y) \rightarrow \exists^{\infty} x \delta(x, y)\right)$ and $p(\pi(y))$ is finite. Then

$$
p(\delta(x, y) \wedge \pi(y))>p(\pi(y)) .
$$

Proof. Clearly $\mathrm{b}(\delta(x, y) \wedge \pi(y)) \geq \mathrm{b}(\pi(y))$. We will prove the sharp inequality by induction. The case $\alpha=0$ being clear we assume that $\delta(x, y)$ and $\pi(y)$ are formulas over some set $A$ and suppose $\mathrm{p}(\pi(y)) \geq \alpha+1$. By definition there is a formula $\theta(y, b)$ p-forking over $A$, such that $\theta(y, b) \vdash \pi(y)$ 
and $\mathrm{p}(\theta(x, b)) \geq \alpha$. As $\models \forall y\left(\theta(y, b) \rightarrow \exists^{\infty} x \delta(x, y)\right)$ the induction hypothesis yields

$$
\mathrm{p}(\theta(y, b) \wedge \delta(x, y))>\mathrm{p}(\theta(y, b))=\alpha .
$$

But $\theta(y, b) \wedge \delta(x, y)$ b-forks over $A$ (because $\theta(y, b) \rightarrow \exists x \delta(x, y))$ so the claim follows.

Proposition 4.8. Assume that $\operatorname{tp}(a / A b)$ b-forks over $A$ and $\operatorname{tp}(a / A b)$ has finite U-rank. Then there are $b^{\prime}, c$ such that $U(\operatorname{tp}(a / A b))=U\left(\operatorname{tp}\left(a / A b b^{\prime} c\right)\right)$ and $\operatorname{tp}\left(a / A b^{\prime} c\right)$ strongly divides over $A c$.

Proof. By definition, there are finitely many formulas $\phi_{i}\left(x, b_{i}\right)$ such that

$$
\operatorname{tp}(a / A b) \vdash \bigvee_{i} \phi_{i}\left(x, b_{i}\right)
$$

and $\phi\left(x, b_{i}\right) \mathrm{p}$-divides over $A$. Finiteness of the U-rank implies that $\operatorname{tp}(a / A b)$ does not fork over $A b$ and therefore $\mathrm{U}\left(\operatorname{tp}(a / A b) \cup\left\{\phi_{m}\left(x, b_{m}\right)\right\}\right)=\mathrm{U}(\operatorname{tp}(a / A b))$ for some $m$. For such $m$, we get that $\operatorname{tp}(a / A b) \cup\left\{\phi_{m}\left(x, b_{m}\right)\right\}$ is a non forking extension of $\operatorname{tp}(a / A b)$; using automorphisms we may assume that $a \models \phi_{m}\left(x, b_{m}\right)$ and $a \downarrow_{A b} b_{m}$.

By definition of $\mathrm{p}$-dividing there is some $c^{\prime}$ such that $\phi\left(x, b_{m}\right)$ strongly divides over $A c^{\prime}$. Let $c \models \operatorname{tp}\left(c^{\prime} / A b b_{m}\right)$ be such that $a \downarrow_{A b b_{m}} c$. Since $c \models$ $\operatorname{tp}\left(c^{\prime} / A b_{m}\right)$ strong dividing is preserved and

$$
\mathrm{U}(\operatorname{tp}(a / A b))=\mathrm{U}\left(\operatorname{tp}\left(a / A b b_{m}\right)\right)=\mathrm{U}\left(\operatorname{tp}\left(a / A b b_{m} c\right)\right)
$$

so letting $b^{\prime}=b_{m}$ proves the proposition.

Proposition 4.9. If a set $\phi(x, b)$ is stable, then there is some $\theta(y) \in \operatorname{tp}(b / \emptyset)$ such that $\phi(x, y) \wedge \theta(y)$ has $N O P$.

Proof.

Claim 4.10. Let $r(y)=\operatorname{tp}(b / \emptyset)$. Then there are no indiscernible sequences $\left\langle a_{i}\right\rangle_{i \in \omega}$ and $\left\langle b_{j}\right\rangle_{j \in \omega}$ such that $b_{j} \models r(y)$ and $\models \phi\left(a_{i}, b_{j}\right)$ if and only if $i \leq j$.

Proof. We may assume without loss of generality (by using automorphisms) that $b_{0}=b$. In this case, $a_{i} \models \phi(x, b)$ which implies that $\phi(x, y) \wedge \phi(x, b)$ witnesses the order property, contradicting Fact 4.4.

Let $p\left(x_{1}, \ldots, x_{n}, \ldots\right), q\left(y_{1}, \ldots, y_{n}, \ldots\right)$ be the (partial) types expressing " $\left\langle x_{i}\right\rangle_{i \in \omega}$ is an indiscernible sequence" and " $\left\langle y_{j}\right\rangle_{j \in \omega}$ is an indiscernible sequence" respectively. By Claim 4.10 the type

$$
p(\bar{x}) \cup q(\bar{y}) \cup\left\{\bigcup_{j} r\left(y_{j}\right)\right\} \cup\left\{\bigcup_{i<j} \phi\left(x_{i}, y_{j}\right)\right\} \cup\left\{\bigcup_{i \geq j} \neg \phi\left(x_{i}, y_{j}\right)\right\}
$$

is inconsistent. By compactness, there is a formula $\theta(y) \in r(y)$ such that 


$$
p(\bar{x}) \cup q(\bar{y}) \cup\left\{\bigcup_{j} \theta\left(y_{j}\right)\right\} \cup\left\{\bigcup_{i<j} \phi\left(x_{i}, y_{j}\right)\right\} \cup\left\{\bigcup_{i \geq j} \neg \phi\left(x_{i}, y_{j}\right)\right\}
$$

is inconsistent and by definition this implies that $\phi(x, y) \wedge \theta(y)$ has NOP.

Corollary 4.11. Let $a, b$ and $c$ be such that there exists $\phi(x, b) \in \operatorname{tp}(a / b c)$ defining a stable set but $U(a / c)=\infty$. Then $a \bigsqcup_{c}^{b} b$.

Proof. Let $a, b, c$ and $\phi(x, b)$ be as in the statement. By Proposition 4.9 there is some $\theta(y) \in \operatorname{tp}(b)$ such that $\psi(x, y):=\phi(x, y) \wedge \theta(y)$ has NOP.

By definition every type extending $\phi(x, b)$ has finite U-rank and $\operatorname{tp}(a / c)$ has infinite U-rank so every completion of $\operatorname{tp}(a / c) \cup\{\phi(x, b)\}$ forks over $c$. This implies that $\psi(x, b)$ forks over $c$. But $\psi(x, y)$ satisfies NOP so Fact 4.3 implies there is a $\psi$-formula witnessing that $\operatorname{tp}(a / b c) \mathrm{p}$-forks over $c$.

4.2. Unstable sets of small dimension. In this section we conclude the proof of Theorem 1.4. The main result which allows us to do this is:

Theorem 4.12. let $T$ be a dependent theory with definable finiteness (i.e. $T$ eliminates the quantifier $\exists^{\infty}$ ) such that the (global) p-ranks of definable sets are bounded by a finite number. Let $\boldsymbol{\Phi}$ be the class of unstable definable sets in $T$, and

$$
n:=\min \{b(\phi(\mathcal{C}, c)) \mid \phi(x, c) \in \mathbf{\Phi}\} .
$$

Let $\phi(x, c) \in \mathbf{\Phi}$ be such that $p(\phi(x, c))=n$ and let $\theta(x, b) \vdash \phi(x, c)$ contain some $a$ with $U(\operatorname{tp}(a / c))=\infty$ and $a \notin \operatorname{acl}(b)$. Then $\theta(x, b)$ is unstable.

Proof. Let $\phi(x, c)$ be an unstable set of minimal p-rank. Assume towards a contradiction that there is a stable set $\theta(x, b)$ and $a \models \theta(x, b)$ satisfying all the assumptions of the theorem. Fix such $\theta(x, b)$ and $a$ for which $m:=$ $\mathrm{U}(\operatorname{tp}(a / c b))$ is maximal. Note that $m$ is well defined as $\mathrm{U}(\operatorname{tp}(a / c b))$, when finite, is bounded by $\mathrm{U}^{\mathrm{b}}(a / \emptyset)$. To simplify the notation we will assume that $c$ is a subsequence of $b$.

To reach a contradiction we will use p-forking to find a definable subset $Y$ of $\phi(x, c)$ consisting of an infinite definable family of almost disjoint copies of $\theta(x, b)$. We can then show that either $I$, the set parametrising the family, is stable which implies that so is $Y$ - contradicting the maximality of $\mathrm{U}(\operatorname{tp}(a / b))$; or $I$ is unstable with $\mathrm{p}(I)<n$, in contradiction to the minimality of $n$.

By Corollary 4.11 we know that $a \chi_{c}^{\mathrm{b}} b$ and by hypothesis $\mathrm{U}(\operatorname{tp}(a / b))=$ $m$. By Proposition 4.8 there are $b_{0}, d$ such that $a \downarrow_{b} b_{0} d$ and $\operatorname{tp}\left(a / b_{0} c d\right)$ contains some $\theta_{0}\left(x, b_{0}\right)$ strongly dividing over $c d$. By definition of strong dividing $\operatorname{tp}\left(b_{0} / c d\right)$ is non algebraic and there is a formula $\pi(y, d)$ such that

$$
\left\{\theta_{0}\left(x, b^{\prime}\right)\right\}_{b^{\prime} \models \pi(y, d)}
$$


is $k$-inconsistent for some $k$. In particular, there are at most $k-1$ elements satisfying $\pi(y, d) \wedge \theta_{0}(a, y)$ so $b_{0}$ is algebraic over $a d$, witnessed by the formula $\theta_{0}(a, y) \wedge \pi(y, d)$.

By assumption $\operatorname{tp}(a / b c)$ is non algebraic and $a \downarrow_{b c} b_{0} d$ so $\operatorname{tp}\left(a / b_{0} d\right)$ is non algebraic and by definable finiteness we know there is a formula $\mu(y) \in$ $\operatorname{tp}\left(b_{0} / c d\right)$ such that $\phi\left(x, b^{\prime}\right)$ is infinite for any $b^{\prime} \models \mu(y)$; we may assume that $\pi(y, d) \rightarrow \mu(y)$.

Claim 4.13. $\operatorname{tp}(a / c d)$ contains no stable definable sets.

Proof. Assume that $\delta(x) \in \operatorname{tp}(a / c d)$ defines a stable set. Since $b_{0} \in \operatorname{acl}(a d)$ there is a formula $\delta(x, y) \in \operatorname{tp}\left(a b_{0} / c d\right)$ such that $\forall x \exists^{<n} y \delta(x, y)$. We may also assume that $\exists y \delta(x, y) \equiv \delta(x)$ so, by Fact 4.6, $\delta(x, y)$ defines a stable set as well.

Since $\mathrm{U}(a / c d) \geq \mathrm{U}(a / b d)$ (recall that $c \subseteq b$ ) the maximality of $m$ implies that in fact $\mathrm{U}(a / c d)=\mathrm{U}(a / b d)$. But $b_{0} \in \operatorname{acl}(a d)$ so $\mathrm{U}\left(a b_{0} / c d\right)=\mathrm{U}(a / c d)=$ $\mathrm{U}(a / b d)$. But $\mathrm{U}\left(a / b d b_{0}\right)=\mathrm{U}(a / b)=m$, so $\mathrm{U}(a / c d)=m$. On the other hand, as $\delta(x, y)$ defines a stable set, we can use Lascar's inequalities to get:

$$
\mathrm{U}\left(a b_{0} / c d\right)=\mathrm{U}\left(b_{0} / c d\right)+\mathrm{U}\left(a / b d b_{0}\right)
$$

But $b_{0} \notin \operatorname{acl}(c d)$ and the last equality implies that $\mathrm{U}(a / c d)>m$, a contradiction.

By Fact 4.6 we know that $\operatorname{tp}\left(a b_{0} / c d\right)$ does not contain any non algebraic formula defining a stable set. In particular, $\pi(\mathcal{C}, d)$ is unstable.

Claim 4.14. $p(\pi(y, d))<p(\phi(x, c))=n$.

Proof. Let $\psi(x, y ; c, d):=\phi(x, c) \wedge \theta_{0}(x, y) \wedge \pi(y, d)$. For all $a^{\prime} \models \phi(x, c)$ there are finitely many $b^{\prime}$ such that $\models \psi\left(a^{\prime}, b^{\prime} ; c, d\right)$; by Fact 4.6, $\mathrm{p}(\psi(x, y ; c, d))=$ $\mathrm{p}(\phi(x, c))=n$.

However, $\theta_{0}\left(x, b^{\prime}\right) \wedge \phi(x, c)$ is non algebraic for any $b^{\prime} \models \pi(y, d)$ so by Fact $4.7 \mathrm{p}(\psi(x, y ; c, d))>\mathrm{p}(\pi(y, d))$.

So $\pi(\mathcal{C}, d)$ is an unstable definable set of p-rank smaller than $n$; this contradicts the minimality of $n$ and the theorem follows.

We can now prove Theorem 1.4;

Corollary 4.15. Let $\mathcal{M}:=(M,<, \ldots)$ an o-minimal structure with elimination of imaginaries and a dense underlying order. Let $\mathcal{N}$ be unstable interpretable in $\mathcal{M}$. Then $\mathcal{N}$ interprets an o-minimal structure.

Proof. Let $\Phi$ be the set of all (non algebraic) unstable $\mathcal{N}$-interpretable sets. For each $Z \in \Phi$ let $(\mathrm{b}(Z), d(Z))$ be the pair consisting of the p-rank and the o-minimal dimension of $Z$. Let $Y \in \Phi$ minimise $(\mathrm{p}(Y), d(Y))$ in the lexicographic order.

Claim 4.16. The o-minimal dimension of $Y$ is 1 . 
Proof. Suppose otherwise. Because $\mathcal{M}$ eliminates imaginaries $Y$ is definable in $\mathcal{M}$. Let $A$ be a set over which $Y$ is definable. Because $Y$ is unstable Theorem 3.8 implies that there is some $Y_{0} \subset Y \mathcal{N}$-definable over $B \supset A$ with $\operatorname{dim} Y_{0}<\operatorname{dim} Y$. Moreover, there exists $a \in Y_{0} \backslash \operatorname{acl}(B)$ such that $\mathrm{U}(\operatorname{tp}(a / A))=\infty$. By Theorem $4.12 Y_{0}$ is unstable so by definition $Y_{0} \in \Phi$ contradicting the minimality of $(\mathrm{p}(Y), d(Y))$.

By Claim 4.16 there is some $Y \in \Phi$ such that the o-minimal dimension of $Y$ is 1 . By Corollary 2.11 $\mathrm{Th}(Y)$ interprets an o-minimal structure, with the desired conclusion.

Recall the following from [OP]:

Definition 4.17. A definable set $\phi(x, a)$ is purely unstable if every definable subset of $\phi(x, a)$ is unstable.

So the last corollary shows, in particular:

Corollary 4.18. Let $\mathcal{M}:=(M,<, \ldots)$ be a dense o-minimal with elimination of imaginaries and $\mathcal{N}$ unstable interpretable in $\mathcal{M}$. Then $\mathcal{N}$ interprets a purely unstable set.

Unfortunately, unlike the results of the previous sections, the present proof does not seem to give significant local data. This is one of the reasons why it is not clear to us, at this stage, what should the right classification of theories interpretable in o-minimal structures look like. The great flexibility in creating local phenomena in o-minimal structures (and to some extent even more so in their reducts) suggests that analysability of types with respect to some "nice" collection of types should be the right direction and the results of this paper suggest that the class of o-minimal (by finite) types has a crucial role in any such analysis. A sharpening of the results of the present section could provide some level of analysis in such terms for weakly unstable types, but the situation in the stable part of the picture is much less obvious.

For types that contain a definable stable set an analysis exists in terms of regular types, hopefully satisfying Zilber's Trichotomy. Such a classification would give a good solution for the class of stably dominated types. However, as the example in Remark 2.12 shows, not all stable types (not even all strongly stable types) contain a formula defining a stable set, or are even stably dominated. The following questions seem natural, and will probably require some additional work:

(1) Is there a (natural) geometric stability theoretic distinction between stable and unstable types (aside from Shelah's combinatorial definition) and between stable and strongly stable types in reducts of o-minimal theories.

(2) What role do stable regular types play in the space of types of a reduct of an o-minimal theory. Do they satisfy Zilber's trichotomy. Can a reasonable theory of analysability be developed in reducts of 
o-minimal theories in terms of regular types and finite by o-minimal types.

(3) Since our local results relate only to types over models, it seems natural to ask whether, in the present context, a reasonable notion of prime models (over arbitrary sets) - an equivalent of a-models in stable theories - exists and what is the right framework for the development of such a theory.

As we pointed out in the introduction, the results of [She] - though not sufficient if one hopes for as sharp results as we would like to obtain - suggest several directions of research that may be of relevance to the above question.

\section{REFERENCES}

[HZ96] Ehud Hrushovski and Boris Zilber. Zariski geometries. Journal of the American Mathematical Society, 9(1):1-55, 1996.

[Joh01] Joseph Johns. An open mapping theorem for o-minimal structures. J. Symbolic Logic, 66(4):1817-1820, 2001.

[Ons02] Alf Onshuus. Th-forking and Rosy Theories. PhD thesis, University of California at Berkeley, 2002.

[Ons06] Alf Onshuus. Properties and consequences of thorn-independence. J. Symbolic Logic, 71(1):1-21, 2006.

[OP] Alf Onshuus and Ya'acov Peterzil. A note on stable sets and groups in theories with nip. To appear in MLQ Math. Log. Q.

[Pi196] Anand Pillay. Geometric stability theory, volume 32 of Oxford Logic Guides. The Clarendon Press Oxford University Press, New York, 1996. Oxford Science Publications.

[PPS00] Ya'acov Peterzil, Anand Pillay, and Sergei Starchenko. Simple algebraic and semialgebraic groups over real closed fields. Trans. Amer. Math. Soc., 352(10):44214450 (electronic), 2000.

[PS98] Ya'acov Peterzil and Sergei Starchenko. A trichotomy theorem for o-minimal structures. Proc. London Math. Soc. (3), 77(3):481-523, 1998.

[She] Saharon Shelah. Dependent theories and the generic pair conjecture. Shelah [Sh:900]. Available on http://shelah.logic.at/short900.html.

[She90] S. Shelah. Classification theory and the number of nonisomorphic models, volume 92 of Studies in Logic and the Foundations of Mathematics. North-Holland Publishing Co., Amsterdam, second edition, 1990.

[She04] Saharon Shelah. Classification theory for elementary classes with the dependence property-a modest beginning. Sci. Math. Jpn., 59(2):265-316, 2004. Special issue on set theory and algebraic model theory.

[vdD98] Lou van den Dries. Tame topology and o-minimal structures, volume 248 of London Mathematical Society Lecture Note Series. Cambridge University Press, Cambridge, 1998.

University of Oxford, Mathematical Institute, 24-29 St Giles', Oxford, OX1 3LB, UK

E-mail address: hasson@maths.ox.ac.uk

Universidad de los Andes, Departemento de Matemáticas, Cra. 1 No 18A10, Bogotá, Colombia

E-mail address: onshuus@gmail.com 